\title{
Communication \\ Catalyst-Free Site Selective Hydroxyalkylation of 5-Phenylthiophen-2-amine with $\alpha$-Trifluoromethyl Ketones through Electrophilic Aromatic Substitution
}

\author{
Valentin Duvauchelle (D), David Bénimélis $\mathbb{D}^{\mathrm{D}}$, Patrick Meffre ${ }^{(D)}$ and Zohra Benfodda *(D)
}

check for

updates

Citation: Duvauchelle, V.;

Bénimélis, D.; Meffre, P.; Benfodda, Z. Catalyst-Free Site Selective

Hydroxyalkylation of

5-Phenylthiophen-2-amine with

$\alpha$-Trifluoromethyl Ketones through

Electrophilic Aromatic Substitution.

Molecules 2022, 27, 925. https://

doi.org/10.3390/molecules27030925

Academic Editors: Graeme Barker

and Simona Rapposelli

Received: 18 January 2022

Accepted: 27 January 2022

Published: 29 January 2022

Publisher's Note: MDPI stays neutral with regard to jurisdictional claims in published maps and institutional affiliations.

Copyright: (c) 2022 by the authors. Licensee MDPI, Basel, Switzerland. This article is an open access article distributed under the terms and conditions of the Creative Commons Attribution (CC BY) license (https:// creativecommons.org/licenses/by/ $4.0 /)$.
UPR CHROME, Université de Nîmes, CEDEX 1, F-30021 Nîmes, France; valentin.duvauchelle@unimes.fr (V.D.); david.benimelis@unimes.fr (D.B.); patrick.meffre@unimes.fr (P.M.)

* Correspondence: zohra.benfodda@unimes.fr

\begin{abstract}
An original and effective approach for achieving trifluoromethyl hydroxyalkylation of 5-phenylthiophen-2-amine using $\alpha$-trifluoromethyl ketones is described. In the last few years, reaction of Friedel-Crafts had been widely used to realize hydroxyalkylation on heterocycles such as indoles or thiophenes by means of Lewis acid as catalyst. Additionally, amine functions are rarely free when carbonyl reagents are used because of their tendency to form imines. This is the first time that a site-selective electrophilic aromatic substitution on $\mathrm{C}_{3}$ atom of an unprotected 5-phenylthiophen-2amine moiety is reported. The liberty to allow reaction in neutral conditions between free amine is valuable in a synthesis pathway. The reaction proceeds smoothly using an atom-economical metaland catalyst-free methodology in good to excellent yields. A mechanism similar to an electrophilic aromatic substitution has been proposed.
\end{abstract}

Keywords: hydroxyalkylation; trifluoromethyl hydroxyalkylation; trifluoromethyl ketone; catalyst-free; site selective; chemoselective; 2-aminothiophene; 5-phenylthiophen-2-amine

\section{Introduction}

2-aminothiophene (2-AT) moiety is widespread in FDA-approved drugs and is a privileged scaffold in medicinal chemistry that is known to confer many biological activities [1-5]. As examples, substituted-2-AT moiety such as compound 1 (Figure 1a) demonstrated activity against Mycobacterium tuberculosis by targeting the Ag85 enzymes [3]. PD 81723 (2, Figure 1a) has been shown to be the first allosteric specific and selective adenosine $\mathrm{A}_{1}$ receptor ligands [6]. Compound 3 has been described as a hepatitis $\mathrm{B}$ virus replication inhibitor [7] and compound 4 showed antimicrobial activity against $A$. fumigatus, G. candidum, C. albicans and S. racemosum [8]. 2-AT derivatives are mostly synthesized using Gewald reaction $[9,10]$. Technically, it involves condensation of a carbonyl derivative, a $\alpha$-cyanoester in the presence of sulfur source. To this day, used methodologies often undergo to the generation of trisubstituted thiophene ring with an electron withdrawing group-particularly negative mesomeric effect-on $C_{3}$ atom (Figure 1a) [11-15].

Over recent decades, interest for hydroxyalkylation on aryl derivatives as $\mathrm{C}-\mathrm{C}$ bond forming reaction has grown [16-19]. Ullyot first reported hydroxyalkylation of aryl compounds with a carbonyl derivative under acidic conditions as new way to synthesize benzoins [20]. Thereafter, synthesis methodologies have been refined to fit with chemical diversity: heteroaryls were used as substrates; carbonyl derivatives were more complex, likewise the Lewis acids. The methodologies described, respectively, by Schnakenburg [21], Ramanathan [22] and Chatti [23] are relevant examples of hydroxyalkylation on heteroaryls scaffolds (Figure $1 b, c$ ).

Hence, trifluoromethyl group introduction onto 2-AT via hydroxyalkylation methodology provides a dual benefit: it introduces a chemical diversity that was lacking in 2-ATs and it inserts the trifluoromethyl group, which is very interesting from a medicinal point of 
view because it is known to confer biological properties of high value [24-27]. Chemists are currently faced with the difficult task of increasing the efficacy of this kind of reactions while also seeking greener processes [28]. As we know, a more responsible use of metals as catalysts as well as the use of atom economical reactions participate in the development of a greener chemistry [29-31]. To our knowledge, the site-selective, metal-and catalyst-free trifluoromethyl hydroxyalkylation of unprotected 5-phenylthiophen-2-amine has never been described in the literature. Herein, we report the trifluoromethyl hydroxyalkylation methodology we developed in presence of various $\alpha$-trifluoromethyl ketones (Figure 1d).

(a) Bioactive 2-aminothiophenes

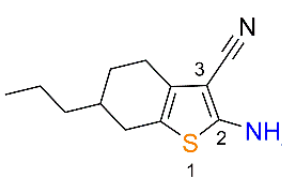

1<smiles>Cc1sc(N)c(C(=O)c2cccc(C(F)(F)F)c2)c1C</smiles>

PD 81723

2

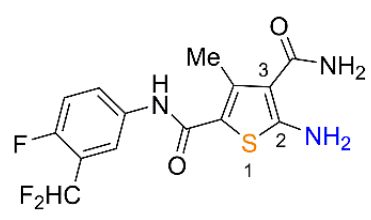

3

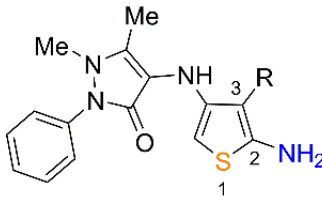

4a: $\mathrm{R}=\mathrm{CN}$ 4b: $\mathrm{R}=\mathrm{CO}_{2} \mathrm{Et}$

(b) Methodology for the access of trifluoromethyl hydroxyalkylated indole attempted on benzothiophene

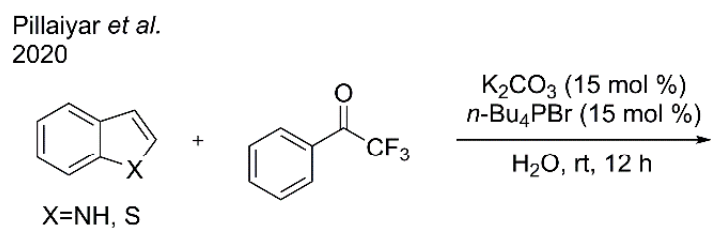

$\mathrm{X}=\mathrm{NH}, \mathrm{S}$

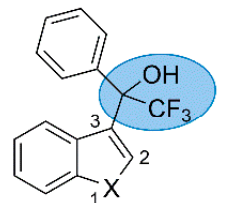

$\mathrm{X}=\mathrm{NH}(\mathbf{9 9} \%) ; \mathrm{S}$ (not obtained)

Unsuccessful trifluoromethyl hydroxyalkylation of benzothiophene on $\mathrm{C}-3$ position

(c) Methodologies for the access of hydroxyalkylated thiophenes

Harikrishnan et al.

2012
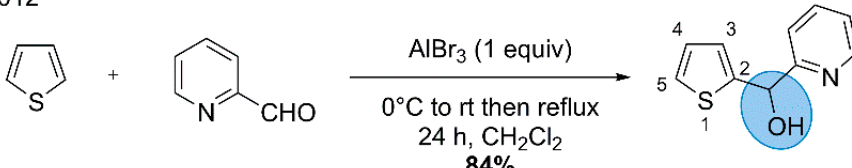

Medimagh et al.

Hydroxyalkylation of thiophene at C-2 position

2011<smiles>[R]C(=O)c1ccc(N([R2])[R])s1</smiles>

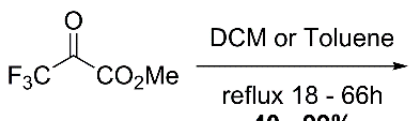

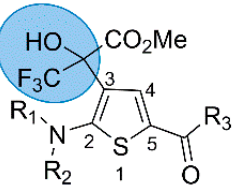

Hydroxyalkylation of thiophene at C-3 position

(d) This work based on former studies and hypothesis

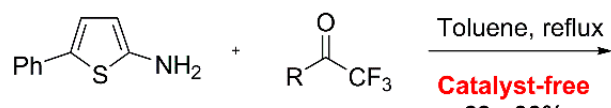

$$
\begin{aligned}
& 69-93 \%
\end{aligned}
$$

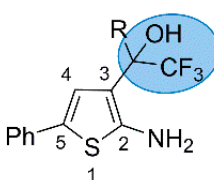

Site selective trifluoromethyl hydroxyalkylation of unprotected 2-aminothiophene

Figure 1. Background and overview. (a) Bioactives 2-AT derivatives. (b) Method to access to trifluoromethyl hydroxyalkylated indole. (c) Methods to access to hydroxyalkylated thiophenes. (d) First hypothesis based on previous studies. 


\section{Results}

To begin, the synthesis of compound 8 has been investigated as described in Scheme 1. 2-bromo-5-nitrothiophene 5 and phenylboronic acid 6, which under Suzuki-Miyaura coupling conditions, developed in our laboratory by Boibessot et al., form intermediate 7 in $86 \%$ yield after purification [32]. Then, we realized the reduction in the nitro function, following Zhang and co-workers methodology, in the presence of hydrazine hydrate in absolute ethanol at $50{ }^{\circ} \mathrm{C}$ for $15 \mathrm{~min}$, followed by the careful addition of Raney nickel to smoothly yield to 8 [33]. (90\% yield after purification).<smiles>O=[N+]([O-])c1ccc(Br)s1</smiles>

5<smiles>Nc1ccc(-c2ccccc2)s1</smiles>

8<smiles>COc1ccccc1</smiles>

6

$\left.1^{\circ}\right) \mathrm{H}_{2} \mathrm{~N}-\mathrm{NH}_{2} \cdot x \mathrm{H}_{2} \mathrm{O}, \mathrm{EtOH}$ $50^{\circ} \mathrm{C}, 15 \mathrm{~min}$

$2^{\circ}$ ) Ni Raney, $50^{\circ} \mathrm{C}, 45 \mathrm{~min}$

$90 \%$
$\mathrm{Pd}\left(\mathrm{PPh}_{3}\right)_{4}, \mathrm{Na}_{2} \mathrm{CO}_{3}[2 \mathrm{M}]$

Toluene/EtOH, reflux, $15 \mathrm{~h}$

$86 \%$

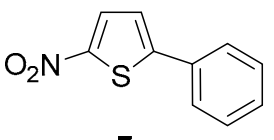

Scheme 1. Synthesis of precursor 8.

On running ${ }^{1} \mathrm{H}$ NMR analysis of compound 8 in $\mathrm{D}_{2} \mathrm{O}$ deuteriation on $\mathrm{C}_{3}$ atom has been observed (Scheme 2). On the ${ }^{1} \mathrm{H}$ spectra, $\mathrm{H}_{3}$ signal disappears and $\mathrm{H}_{4}$ signal appears as a singlet at $7.28 \mathrm{ppm}$. On ${ }^{13} \mathrm{C}$ spectra, $\mathrm{C}_{3}$ atom couples with the deuterium it carries to give a triplet at 123.40 ppm (See Supplementary Materials for More Details, Figures S1 and S2).
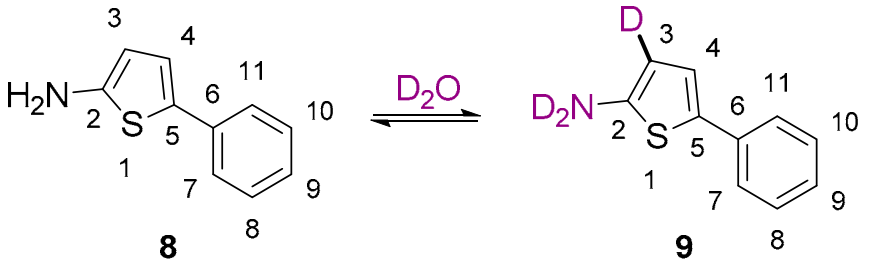

Scheme 2. Deuteriation of 8 on $\mathrm{C}_{3}$ atom in presence of deuterium oxide.

The deuteriation on $\mathrm{C}_{3}$ atom seems to be due to the positive mesomeric $(+\mathbf{M})$ effect of the amino group responsible for the reactivity shown thereafter. ${ }^{17}$ A plausible mechanism of this deuteriation is proposed in Scheme 3, such as suggested by Garnett and his team [34]. A delocalization of the lone pair of nitrogen atoms would result in a deuteriation on $C_{3}$ atom to generate the intermediate $\mathbf{1 0}$ before the rearomatization of the structure to afford $\mathbf{9}$.

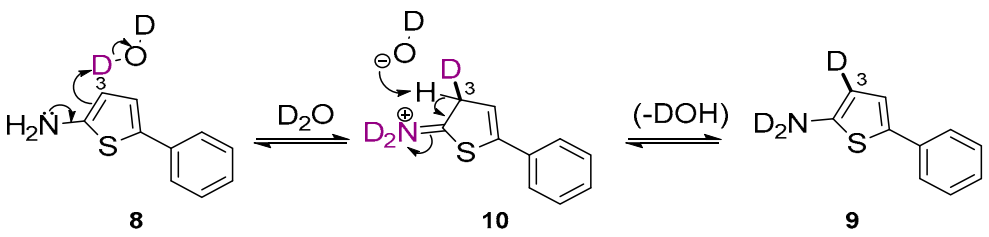

Scheme 3. Proposed mechanism for the deuteriation of 9.

The reactivity of $\mathbf{8}$ was then studied in the presence of other electrophiles. When $\mathbf{8}$ is reacted with $p$-anisaldehyde or acetophenone in toluene under reflux, no reaction occurs and only starting materials are recovered (See Supplementary Materials, Table S1). Facing this lack of reactivity, we decided to use stronger electrophilic compounds to exploit the natural reactivity of 2 -AT. $\alpha$-trifluorinated ketones have been chosen, as suggested in the literature [35]. In that case, when $\mathbf{8}$ is in the presence of $\alpha$-trifluorinated ketone 11e in toluene under reflux, substitution product 12e is obtained in good yields. It suggests that $\alpha$-trifluomethyl ketone are harder electrophiles than methyl ketones, following the hard 
and soft acids and bases theory [36,37]. Temperature has been investigated. Best yield of $83 \%$ has been obtained when temperature was set to $120^{\circ} \mathrm{C}$. At $100^{\circ} \mathrm{C}$, the reaction was incomplete and decomposition products have been observed at $140{ }^{\circ} \mathrm{C}$ (Table 1 , entries $1-3)$. When an excess of $\alpha$-trifluorinated ketone 11 e (1.5 and 2.0 equiv.) was reacted with 8 , yields stayed similar (Table 1, entries 4-5).

Table 1. Optimization studies for the synthesis of $\mathbf{1 2 e}$.

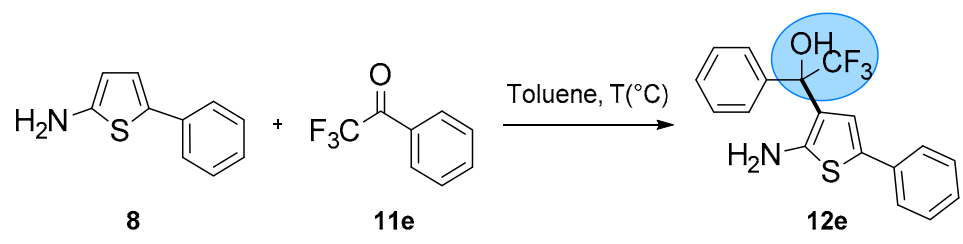

\begin{tabular}{cccc}
\hline Entry & 11e Equivalent (eq.) & T ( $\left.{ }^{\circ} \mathbf{C}\right)$ & Yield (\%) $^{\mathbf{b}}$ \\
\hline $1^{\mathrm{a}}$ & 1 & 100 & $70 \%$ \\
$2^{\mathrm{a}}$ & 1 & 120 & $83 \%$ \\
$3^{\mathrm{a}}$ & 1 & 140 & $47 \%$ \\
$4^{\mathrm{a}}$ & 1.5 & 120 & $82 \%$ \\
$5^{\mathrm{a}}$ & 2 & 120 & $81 \%$ \\
\hline
\end{tabular}

a Experiment conducted in toluene. ${ }^{b}$ Yields obtained after purification on flash chromatography.

The reaction occurs under metal and catalyst free conditions, in toluene under reflux for 2 to $5 \mathrm{~h}$. As predicted, the reaction is site selective. This reactivity may be directed by the $+\mathbf{M}$ effect of the amino group, which confers an enhanced nucleophilic reactivity of the $\mathrm{C}_{3}$ atom and allows reaction with $\alpha$-trifluoromethyl ketones as electrophiles. When acidic catalytic conditions are used $\left(\mathrm{AlCl}_{3}\right.$ or $\left.\mathrm{Sc}(\mathrm{OTf})_{3}\right)$ no product is observed, showing that the donor effect of the amino group is sufficient to observe the formation of the desired derivative (See Supplementary Materialsfor more details, Table S2).

Additionally, when the amino group is replaced by the electron withdrawing nitro group on 7, no substitution product is formed in presence of ketone 11e. Whether in the presence or absence of acidic catalysis $\left(\mathrm{AlCl}_{3}\right.$ and $\mathrm{Sc}(\mathrm{OTf})_{3} 10 \mathrm{~mol} \%$, see Supplementary Materials for More Details, Table S3), only the starting material has been recovered showing that the amine function is important. The presence of $+\mathbf{M}$ effect of the amino function is crucial in the reactivity (Scheme 4).<smiles>O=[N+]([O-])c1ccc(-c2ccccc2)s1</smiles>

7<smiles>O=C(c1ccccc1)C(F)(F)F</smiles>

$11 \mathrm{e}$

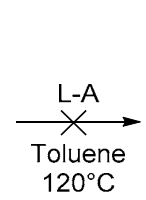

$120^{\circ} \mathrm{C}$

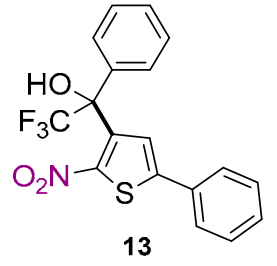

not formed

Scheme 4. Attempts of hydroxyalkylation of 7.

To investigate the scope of the proposed methodology, various $\alpha$-trifluoromethyl ketones 11a-o were allowed to react with 5-phenylthiophen-2-amine 8 in stoechiometric amounts in toluene under reflux for 2 to $4.5 \mathrm{~h}$ (Scheme 5). The aliphatic nature of the group grafted on ketone did not prevent the reactivity and substitution molecules are formed in good yields (12a: 87\%, 12b: 81\%). Aryls and heteroaryls groups were also investigated and good to excellent yields were obtained with 6-membered rings (12e-j: 75-93\%), giving slightly better yields than 5-membered rings (12c: 76\%, 12d: 69\%). Steric hindrance did not seem to be a determining factor because aromatic bicycles reacted smoothly to afford desired compounds in very good yields too (12k: 80\%, 121: 72\%). In most cases, the hydroxyalkylation was observed in good to excellent yields after purification with flash chromatography (69-93\%). Reaction did not occur in the presence of ketones 11m, 11n and 11o. Anyway, this reaction seems to be substrate-dependent, in light of the absence of 
reactivity for $\alpha$-trifluoromethyl ketones $\mathbf{1 1} \mathbf{m}-\mathbf{o}$. The presence of pyrrole and indole, known to be two rich electron heterocycles, may be responsible for the deactivation of the hard nucleophilic center that is the trifluoromethyl ketones 11n and 11o [38]. Considering the perfluoro-2-hexanone $11 \mathrm{~m}$, no example of such reactivity has been reported in the literature for the last 20 years (see Supplementary Materials for More Details, Table S4).

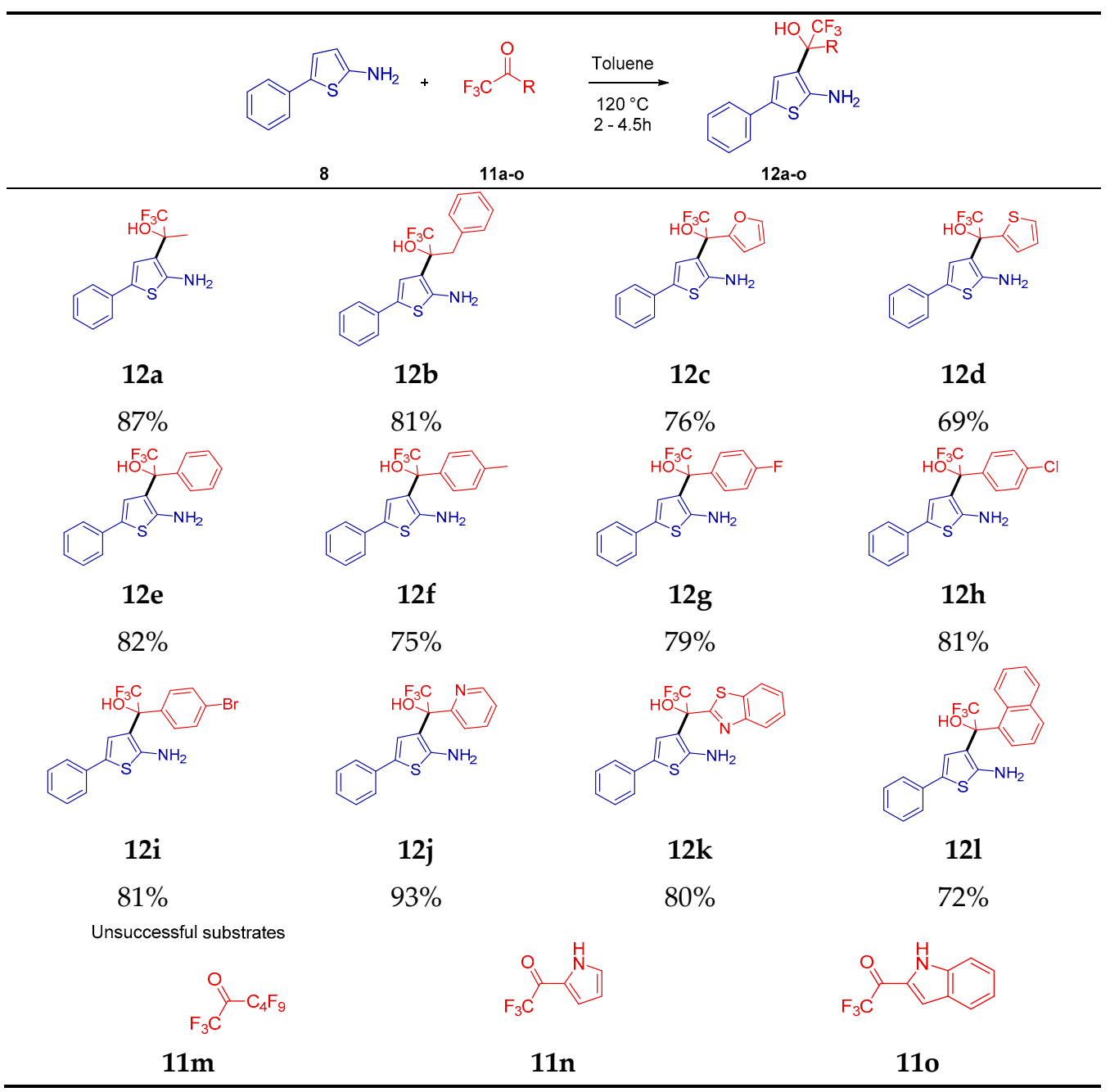

Scheme 5. Scope of substituted aminothiophenes. Reactions conditions: 5-phenylthiophen-2-amine 8 (1.0 equiv.), $\alpha$-trifluoromethyl ketones 11a-o (1.0 equiv.), toluene $(3.5 \mathrm{~mL} / \mathrm{mmol})$, under argon for $2-4.5 \mathrm{~h}, 120^{\circ} \mathrm{C}$. Yields obtained after purification on flash chromatography. Not isolated, only starting materials have been recovered.

A mechanistic proposal is given in Scheme 6 for the conversion of 8 into 15 through a similar mechanism of an electrophilic aromatic substitution [39-41]. First step is the attack of $C_{3}$ atom on electrophilic center of trifluoromethyl ketone to afford intermediate 14 . Then, aromatization drives the formation of structure $\mathbf{1 5}$.<smiles>[R]C(=O)CCCCC(N)=O</smiles>

8<smiles>[R]C([R])(F)C(CO)(CO)c1cc(-c2ccccc2)sc1N</smiles>

14<smiles></smiles>

15

Scheme 6. Mechanistic proposal for the coupling reaction with 5-phenyl-2-aminothiophene 8 and $\alpha$-trifluoromethyl ketone. 
An X-ray crystal structure was carried out to establish the authenticity of $\mathbf{1 2} \mathbf{j}$ structure. We can observe that the structure has a planar part composed of the phenyl and thiophenyl moiety. Then, alkylation observed and assessed on $C_{2}$ atom is composed of a pyridyl group almost perpendicular to both other aromatic cycles. A very strong intramolecular $\mathrm{H}$-bond is observed between the new hydroxyl generated group and the amine group of the thiophenyl moiety $\left[\mathrm{N}_{1}-\mathrm{H}_{1 \mathrm{c}} \cdots \mathrm{O}_{1} 2.187 \AA\right.$ and $\left.129^{\circ}\right]$ (Figure 2a,b). This bond, stabilizing the whole structure, could drive the reaction. Another one is observed between nitrogen atom of pyridyl group and hydroxyl function $\left[\mathrm{O}_{1}-\mathrm{H}_{1 \mathrm{c}} \cdots \mathrm{N}_{2} 2.237 \AA\right.$ and $\left.137^{\circ}\right]$. (See Supplementary Materials, Figures S3 and S4, Tables S5-S9). The expansion of the packing diagram also showed the alternance of $\mathrm{R}$ and $\mathrm{S}$ enantiomers in the crystal mesh. (Figure $2 b$ ). Since no catalysts or chiral auxiliaries were used, we did not expect the reaction to be enantioselective.

(a)
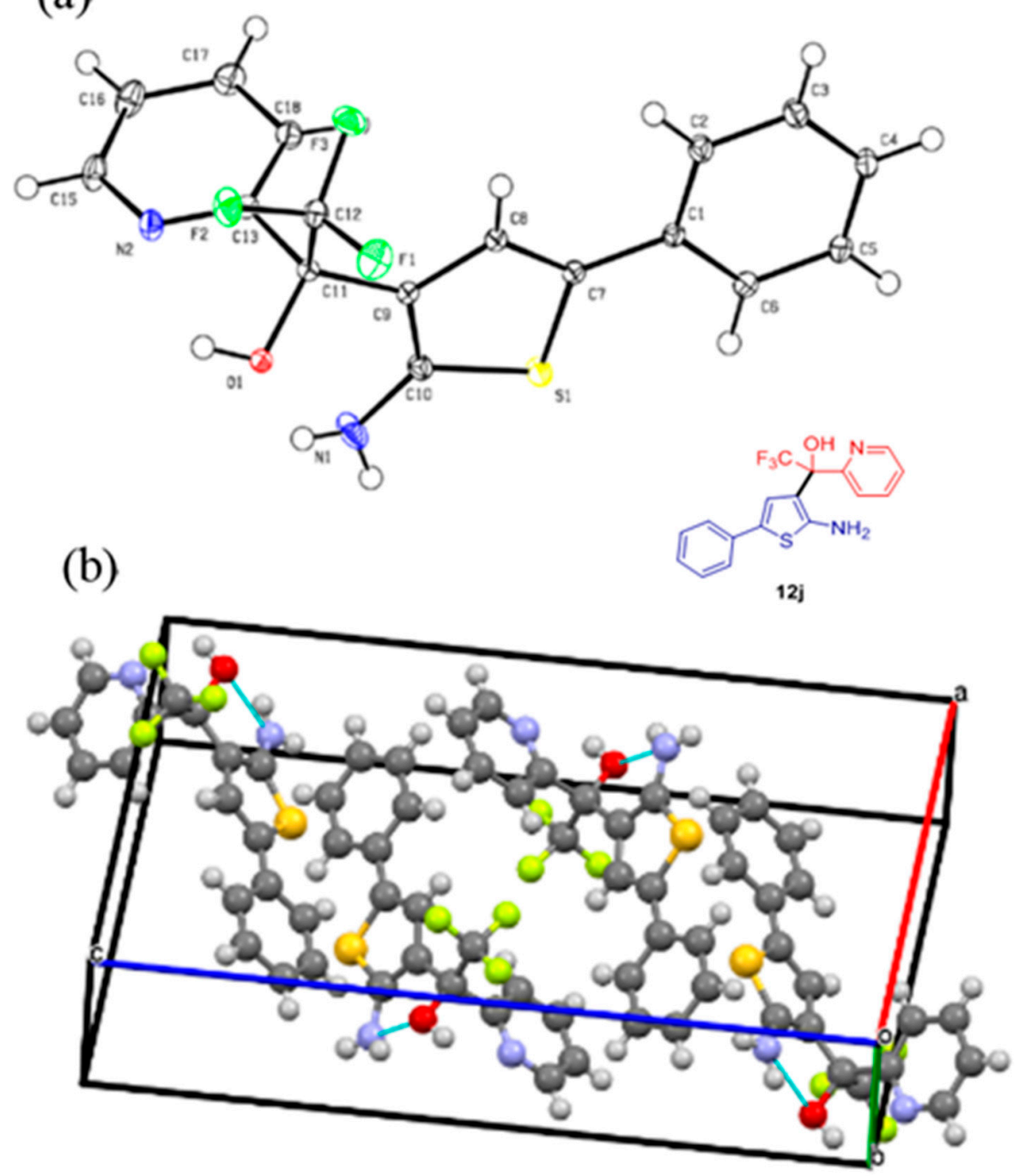

Figure 2. (a) XP diagram of compound 12j with atomic numbering scheme; (b) Expansion of the packing diagram of compound $\mathbf{1 2} \mathbf{j}$ within the crystal mesh trough intra- and intermolecular hydrogen bonds.

\section{Conclusions}

In summary, we have developed an atom-economical approach to synthesize sitespecific substituted 5-phenylthiophen-2-amine from simple and commercially available starting materials, namely, $\alpha$-trifluoromethyl ketones and 5-phenylthiophen-2-amine, by exploiting a trifluoromethyl hydroxyalkylation reaction. The chosen scope shows the 
variety we can introduce on 5-phenylthiophen-2-amine scaffold. The reaction showed good to excellent yields after purification (69-93\%) and with a total chemoselectivity given that only carbon-carbon bond is formed. The site selective introduction of trifluoromethyl hydroxyalkyl groups contrasts with traditionally inserted substituents with -M effect on thiophene scaffold. Moreover, the chemo-and regioselectivity described allows flexibility in substitutions possibilities in drug discovery.

\section{Materials and Methods}

\subsection{General Experimental Methods}

All reagents were purchased from commercial suppliers (Acros Geel-Belgium, Sigma Aldrich L'lsle-d'Abeau Chesnes_-France, Alfa Aesar Kandel-Germany and TCI ZwijndrechtBelgium) and were used without further purification. NMR spectra were recorded with a Bruker Avance 300 spectrometer (300 MHz and $75 \mathrm{MHz}$ for ${ }^{1} \mathrm{H}$ and ${ }^{13} \mathrm{C}$ NMR, respectively) and Bruker Avance 400 spectrometer $\left(376.5 \mathrm{MHz}\right.$ for $\left.{ }^{19} \mathrm{~F}\right)$. Chemical shifts $(\delta)$ and coupling constants $(J)$ are given in ppm and $\mathrm{Hz}$, respectively, using residual solvent signals as reference for the ${ }^{1} \mathrm{H}$ and ${ }^{13} \mathrm{C}$. The following abbreviations are used: $\mathrm{s}=$ singlet, $\mathrm{d}=\mathrm{doublet}$, $\mathrm{t}=$ triplet, $\mathrm{q}=$ quartet, br $\mathrm{s}=$ broad signal, $\mathrm{dd}=$ doublet of doublets, $\mathrm{dt}=$ double of triplets, $\mathrm{m}=$ multiplet. High-resolution mass spectra (HRMS) were obtained by electrospray using a TOF analyzer Platform. IR spectra were obtained using a Jasco FT-IR 410 instrument as a thin film on $\mathrm{NaCl}$ disc as stated; only structurally important peaks $(\bar{v})$ are presented in $\mathrm{cm}^{-1}$. Reactions were monitored with Merck Kieselgel $60 \mathrm{~F}_{254}$ precoated aluminum silica gel plates (0.25 mm thickness). Melting points were determined on a Stuart scientific SMP10 apparatus and are uncorrected. Flash chromatography was performed on a Grace Reveleris X2 using a $40 \mu \mathrm{m}$ packed silica cartridge. HPLC analyses were obtained on the Waters Alliance 2795 using the following conditions: Thermo Hypersil C18 column ( $3 \mu \mathrm{m}$, $50 \mathrm{~mm} \times 2.1 \mathrm{~mm}), 20{ }^{\circ} \mathrm{C}$ column temperature, $0.2 \mathrm{~mL} / \mathrm{min}$ flow rate, photodiodearray detection (210-400 nm), mobile phase consistent of a gradient of water and acetonitrile (each containing $0.1 \%$ trifluoroacetic acid). UPLC analyses were obtained on the Waters Acquity H-Class using the following conditions: Waters Acquity BEH C18 column $(1.7 \mu \mathrm{m}$, $50 \times 2.1 \mathrm{~mm}), 25{ }^{\circ} \mathrm{C}$ column temperature, $0.5 \mathrm{~mL} / \mathrm{min}$ flow rate, photodiodearray detection (TUV-214 nm), mobile phase consistent of a gradient of water and acetonitrile (each containing $0.1 \%$ of formic acid).

\subsection{Preparation of 5-Phenylthiophen-2-amine (8)}

Starting from 2-bromo-5-nitrothiophene 5 (2.4 mmol; $500 \mathrm{mg})$, phenylboronic acid 6 $(3.6 \mathrm{mmol} ; 440 \mathrm{mg})$ and $\mathrm{Pd}\left(\mathrm{PPh}_{3}\right)_{4}(0.12 \mathrm{mmol} ; 140 \mathrm{mg})$ in a mixture of toluene/ethanol $(16 \mathrm{~mL}, 2.3: 1, v / v)$ was added a $[2 \mathrm{M}]$ of aqueous solution of $\mathrm{Na}_{2} \mathrm{CO}_{3}(4.8 \mathrm{mmol} ; 4.5 \mathrm{~mL})$. The reaction mixture was refluxed over $15 \mathrm{~h}$. The cold solution was diluted with ethyl acetate $(50 \mathrm{~mL})$ and filtered through a Celite pad, and the filtrate was diluted with water $(60 \mathrm{~mL})$. The aqueous solution was extracted with ethyl acetate $(3 \times 50 \mathrm{~mL})$. The organic phases were combined, dried over MgSO4, filtered, and concentrated under reduced pressure to give the crude compound. The residue was purified by flash column chromatography (silica gel, AcOEt/Petroleum ether (PE), 0/100 ramping to 100/0, v/v) to give the desired compound. 2-nitro-5-phenylthiophene (7): Yield: 86\% (756 mg); yellow powder: $\mathrm{mp} 124-126^{\circ} \mathrm{C}$ (lit. [32] 123-124 ${ }^{\circ} \mathrm{C}$ ); $\mathrm{R}_{f}$ : 0.51 (PE/AcOEt: 8/2). $v_{\max } / \mathrm{cm}^{-1}$ 1512 (N-O). ${ }^{1} \mathrm{H}$ NMR (300 MHz, DMSO-d6) $\delta 7.47-7.55\left(\mathrm{~m}, 3 \mathrm{H}, 3 \mathrm{H}_{\mathrm{Ar}}\right), 7.66-7.55(\mathrm{~m}, 1 \mathrm{H}$, $\left.\mathrm{H}_{\mathrm{Ar}}\right), 7.80-7.87\left(\mathrm{~m}, 2 \mathrm{H}, 2 \mathrm{H}_{\mathrm{Ar}}\right), 8.17\left(\mathrm{~d}, 1 \mathrm{H}, \mathrm{J}=4.5 \mathrm{~Hz}, \mathrm{H}_{\mathrm{Ar}}\right) .{ }^{13} \mathrm{C} \mathrm{NMR}\left(\mathrm{DMSO}-\mathrm{d}_{6}, 75 \mathrm{MHz}\right) \delta$ 124.07 $\left(\mathrm{CH}_{\mathrm{Ar}}\right), 126.29\left(2 \mathrm{CH}_{\mathrm{Ar}}\right), 129.54\left(2 \mathrm{CH}_{\mathrm{Ar}}\right), 130.35\left(\mathrm{CH}_{\mathrm{Ar}}\right), 131.37\left(\mathrm{CH}_{\mathrm{Ar}}\right), 131.48\left(\mathrm{C}_{\mathrm{Ar}}\right)$, $149.32\left(\mathrm{C}_{\mathrm{Ar}}\right), 151.52\left(\mathrm{C}-\mathrm{NO}_{2}\right)$. UPLC: $\mathrm{t}_{\mathrm{R}}$ : $3.15 \mathrm{~min}$; purity: 97\%; HRMS: $[\mathrm{M}+\mathrm{H}]^{+}$calculated for $\mathrm{C}_{10} \mathrm{H}_{8} \mathrm{NO}_{2}$ S: 206.0276; found: 206.0276 .

The synthesis of 5-phenylthiophen-2-amine (8) was prepared according to a procedure described by Zhang et al. or with minor modifications thereof [33]. To a solution of 2-nitro-5-phenylthiophene (7) (1.71 mmol; $300 \mathrm{mg})$ and Pd/C (0.28 mmol; $30 \mathrm{mg})$ in absolute ethanol $(5 \mathrm{~mL}, \mathrm{C}=0.1 \mathrm{M})$ was added hydrazine hydrate $(15 \mathrm{eq})$. The reaction 
was stirred at $50{ }^{\circ} \mathrm{C}$ for $20 \mathrm{~min}$ and an excess of Raney nickel slurry in water (1.2 eq) was slowly added. The reaction was monitored by TLC (PE/AcOEt 70/30). After $1.5 \mathrm{~h}$, when the bubbling ceased, the mixture was cooled to room temperature and filtered through celite. The filtrate was condensed under reduced pressured and the crude was purified on flash silica gel chromatography (PE/AcOEt 100/0 ramping 0/100 v/v) to afford the desired product. 5-phenylthiophen-2-amine (8): Yield: 90\% (232 mg); white powder: $\mathrm{mp}$ 127-129 ${ }^{\circ} \mathrm{C}$. $\mathrm{R}_{\mathrm{f}}$ : 0.64 (PE/EtOAc: 70/30). $v_{\max } / \mathrm{cm}^{-1} 3110$ (NH). ${ }^{1} \mathrm{H}$ NMR $(300 \mathrm{MHz}$, DMSO-d6) $\delta 5.77$ (br s, 2H, $\left.\mathrm{NH}_{2}\right), 5.86\left(\mathrm{~d}, J=3.8 \mathrm{~Hz}, 1 \mathrm{H}, \mathrm{H}_{\mathrm{Ar}}\right), 7.01\left(\mathrm{~d}, J=3.8 \mathrm{~Hz}, 1 \mathrm{H}, \mathrm{H}_{\mathrm{Ar}}\right)$, 7.05-7.12 (m, 1H, $\left.\mathrm{H}_{\mathrm{Ar}}\right), 7.24-7.31\left(\mathrm{~m}, 1 \mathrm{H}, \mathrm{H}_{\mathrm{Ar}}\right), 7.35-7.41\left(\mathrm{~m}, 1 \mathrm{H}, \mathrm{H}_{\mathrm{Ar}}\right) .{ }^{1} \mathrm{H}$ NMR $(300 \mathrm{MHz}$, $\left.\mathrm{D}_{2} \mathrm{O}\right) \delta 7.30\left(\mathrm{~s}, 1 \mathrm{H}, \mathrm{H}_{\mathrm{Ar}}\right), 7.38-7.50\left(\mathrm{~m}, 3 \mathrm{H}, 3 \mathrm{H}_{\mathrm{Ar}}\right), 7.64-7.69\left(\mathrm{~m}, 2 \mathrm{H}, 2 \mathrm{H}_{\mathrm{Ar}}\right) .{ }^{13} \mathrm{C} \mathrm{NMR}$ $\left(75 \mathrm{MHz}, \mathrm{DMSO}-\mathrm{d}_{6},\right) \delta 104.72\left(\mathrm{CH}_{\mathrm{Ar}}\right), 123.05\left(\mathrm{CH}_{\mathrm{Ar}}\right), 123.39\left(2 \mathrm{CH}_{\mathrm{Ar}}\right), 125.01\left(\mathrm{C}_{\mathrm{Ar}}\right), 125.08$ $\left(\mathrm{CH}_{\mathrm{Ar}}\right), 128.80\left(2 \mathrm{CH}_{\mathrm{Ar}}\right), 135.04\left(\mathrm{C}_{\mathrm{Ar}}\right), 154.51\left(\mathrm{C}-\mathrm{NH}_{2}\right) .{ }^{13} \mathrm{C} \mathrm{NMR}\left(75 \mathrm{MHz}, \mathrm{D}_{2} \mathrm{O}\right) \delta 122.06$ $\left(\mathrm{CH}_{\mathrm{Ar}}\right), 123.40\left(\mathrm{t}, J=15.7 \mathrm{~Hz}, \mathrm{CD}_{\mathrm{Ar}}\right), 125.53\left(2 \mathrm{CH}_{\mathrm{Ar}}\right), 128.48\left(\mathrm{CH}_{\mathrm{Ar}}\right), 129.07\left(\mathrm{C}_{\mathrm{Ar}}\right), 129.22$ $\left(2 \mathrm{CH}_{\mathrm{Ar}}\right), 132.67\left(\mathrm{CH}_{\mathrm{Ar}}\right), 142.24\left(\mathrm{C}-\mathrm{NH}_{2}\right)$. UPLC: $\mathrm{t}_{\mathrm{R}}$ : 3.15 min; purity: $97 \%$; HRMS: $[\mathrm{M}+\mathrm{H}]^{+}$ calcd for $\mathrm{C}_{10} \mathrm{H}_{10} \mathrm{NS}$ : 176.0528 ; found: 176.0527 .

\subsection{Procedure for the Preparation of Trifluorohydroxyalkyl-5-Phenylthiophen-2-amine (12a-121)}

In a round bottom flask, 5-phenylthiophen-2-amine (8) (1eq, $0.57 \mathrm{mmol} ; 100 \mathrm{mg}$ ), and the corresponding trifluorinated compound (12a-o) (1eq, $0.57 \mathbf{m m o l}$ ) are added in $2 \mathrm{~mL}$ of dry toluene under reflux and inert atmosphere until complete substrate consumption followed by TLC (PE/AcOEt 7/3) and HPLC $\left(\mathrm{H}_{2} \mathrm{O} / \mathrm{ACN}\right)$. The cold solution is then diluted with AcOEt $(50 \mathrm{~mL})$ and water $(50 \mathrm{~mL})$. The aqueous layer is extracted with ethyl acetate $(3 \times 50 \mathrm{~mL})$ and the combined organic layers are washed with water $(50 \mathrm{~mL})$, brine $(50 \mathrm{~mL})$, dried over $\mathrm{MgSO}_{4}$ and concentrated under reduced pressure to give the crude compound (brown oil). The residue is further purified by flash column chromatography (silica gel, AcOEt/PE, 0/100 ramping to 100/0,v/v) to give the desired compound as a brown powder or brown crystals.

2-(2-amino-5-phenylthiophen-3-yl)-1,1,1-trifluoropropan-2-ol (12a): Yield: 87\% (142 mg); brown powder: $\mathrm{mp} 135-137^{\circ} \mathrm{C} . \mathrm{R}_{f}: 0.44$ (PE/AcOEt: $\left.70 / 30 \mathrm{v} / \mathrm{v}\right) \cdot v_{\max } / \mathrm{cm}^{-1} 3615(\mathrm{NH})$, $3362(\mathrm{OH}), 1609(\mathrm{~N}-\mathrm{H})$ and $1144(\mathrm{C}-\mathrm{OH}) .{ }^{1} \mathrm{H}$ NMR $(300 \mathrm{MHz}, \mathrm{DMSO}-\mathrm{d} 6) \delta 1.67$ (s, 3H, $\left.\mathrm{CH}_{3}\right), 5.87$ (br s, 2H, NH $\left.\mathrm{NH}_{2}\right), 6.72\left(\mathrm{~s}, 1 \mathrm{H}, \mathrm{H}_{\mathrm{Ar}}\right), 7.03(\mathrm{~s}, 1 \mathrm{H}, \mathrm{OH}), 7.12\left(\mathrm{t}, J=7.3 \mathrm{~Hz}, 1 \mathrm{H}, \mathrm{H}_{\mathrm{Ar}}\right)$, $7.29\left(\mathrm{t}, J=7.5 \mathrm{~Hz}, 2 \mathrm{H}, 2 \mathrm{H}_{\mathrm{Ar}}\right), 7.40\left(\mathrm{~d}, J=8.1 \mathrm{~Hz}, 2 \mathrm{H}, 2 \mathrm{H}_{\mathrm{Ar}}\right) .{ }^{13} \mathrm{C}$ NMR $(75 \mathrm{MHz}, \mathrm{DMSO}-\mathrm{d} 6)$ $\delta 23.11\left(\mathrm{CH}_{3}\right), 73.87\left(\mathrm{q},{ }^{2} J_{\mathrm{CF}}=28.6 \mathrm{~Hz}, \mathrm{C}-\mathrm{CF}_{3}\right), 113.42\left(\mathrm{C}_{\mathrm{Ar}}\right), 122.89\left(\mathrm{C}_{\mathrm{Ar}}\right), 123.17\left(\mathrm{CH}_{\mathrm{Ar}}\right)$, $123.48\left(2 \mathrm{CH}_{\mathrm{Ar}}\right), 125.49\left(\mathrm{CH}_{\mathrm{Ar}}\right), 126.60\left(\mathrm{q},{ }^{1} J_{\mathrm{CF}}=285.8 \mathrm{~Hz}, \mathrm{CF}_{3}\right), 128.84\left(2 \mathrm{CH}_{\mathrm{Ar}}\right), 134.43\left(\mathrm{C}_{\mathrm{Ar}}\right)$, $152.47\left(\mathrm{C}_{\mathrm{Ar}}\right) .{ }^{19} \mathrm{~F}$ NMR $(376 \mathrm{MHz}, \mathrm{DMSO}-\mathrm{d} 6) \delta-80.50\left(\mathrm{CF}_{3}\right)$. HPLC: $\mathrm{t}_{\mathrm{R}}: 30.28 \mathrm{~min}$. HRMS: $[\mathrm{M}+\mathrm{H}]^{+}$calcd for $\mathrm{C}_{13} \mathrm{H}_{13} \mathrm{NOSF}_{3}$ : 288.0670; found: 288.0685 .

2-(2-amino-5-phenylthiophen-3-yl)-1,1,1-trifluoro-3-phenylpropan-2-ol (12b): Yield: 81\% (168 mg); brown powder: mp 118-119 ${ }^{\circ} \mathrm{C}$. $\mathrm{R}_{f}$ : 0.37 (PE/AcOEt: 70/30 v/v). $v_{\max } / \mathrm{cm}^{-1}$ $3615(\mathrm{NH}), 3375(\mathrm{OH}), 1596(\mathrm{~N}-\mathrm{H})$ and $1145(\mathrm{C}-\mathrm{OH}) .{ }^{1} \mathrm{H}$ NMR (300 MHz, DMSO-d6) $\delta$ $3.16\left(\mathrm{~d}, J=14.2 \mathrm{~Hz}, 1 \mathrm{H}, \mathrm{CH}_{2 \mathrm{a}}\right), 3.51\left(\mathrm{~d}, J=14.2 \mathrm{~Hz}, 1 \mathrm{H}, \mathrm{CH}_{2 \mathrm{~b}}\right), 5.71\left(\mathrm{br} \mathrm{s}, 2 \mathrm{H}, \mathrm{NH}_{2}\right)$, $6.92\left(\mathrm{~s}, 1 \mathrm{H}, \mathrm{H}_{\mathrm{Ar}}\right), 7.08-7.21\left(\mathrm{~m}, 5 \mathrm{H}, 5 \mathrm{H}_{\mathrm{Ar}}\right), 7.26-7.35\left(\mathrm{~m}, 4 \mathrm{H}, 3 \mathrm{H}_{\mathrm{Ar}}, \mathrm{OH}\right), 7.37-7.42(\mathrm{~m}, 2 \mathrm{H}$, $2 \mathrm{H}_{\mathrm{Ar}}$ ). The signal corresponding to $\mathrm{CH}_{2}$ appears under the solvent signal on ${ }^{13} \mathrm{C}$ spectrum. Yet, the signal appears on DEPT-135 spectrum. ${ }^{13} \mathrm{C}$ NMR (75 MHz, DMSO-d6) $\delta$ $38.82\left(\mathrm{CH}_{2}\right), 78.04\left(\mathrm{q}^{2} \mathrm{~J}_{\mathrm{CF}}=27.2 \mathrm{~Hz}, \mathrm{C}-\mathrm{CF}_{3}\right), 110.57\left(\mathrm{C}_{\mathrm{Ar}}\right), 120.78\left(\mathrm{C}_{\mathrm{Ar}}\right), 122.90\left(\mathrm{C}_{\mathrm{Ar}}, \mathrm{CH}_{\mathrm{Ar}}\right)$, $123.49\left(2 \mathrm{CH}_{\mathrm{Ar}}\right), 125.49\left(2 \mathrm{CH}_{\mathrm{Ar}}\right), 126.26\left(\mathrm{CH}_{\mathrm{Ar}}\right), 126.43\left(\mathrm{q},{ }^{1} J_{\mathrm{CF}}=287.2 \mathrm{~Hz}, \mathrm{CF}_{3}\right), 127.48$ $\left(2 \mathrm{CH}_{\mathrm{Ar}}\right), 128.85\left(2 \mathrm{CH}_{\mathrm{Ar}}\right), 130.83\left(2 \mathrm{CH}_{\mathrm{Ar}}\right), 134.46\left(\mathrm{C}_{\mathrm{Ar}}\right), 135.34\left(\mathrm{C}_{\mathrm{Ar}}\right), 152.85\left(\mathrm{C}_{\mathrm{Ar}}\right) .{ }^{19} \mathrm{~F} \mathrm{NMR}$ (376 MHz, DMSO-d6) $\delta-76.34\left(\mathrm{CF}_{3}\right)$. HPLC: $\mathrm{t}_{\mathrm{R}}: 32.13 \mathrm{~min}$. HRMS: $[\mathrm{M}+\mathrm{H}]^{+}$calcd for $\mathrm{C}_{19} \mathrm{H}_{17} \mathrm{~F}_{3} \mathrm{NS}$ : 364.0977; found: 364.0982.

1-(2-amino-5-phenylthiophen-3-yl)-2,2,2-trifluoro-1-(furan-2-yl)ethan-1-ol (12c): Yield: 76\% (147 mg); brown powder: $\mathrm{mp} 126-128^{\circ} \mathrm{C}$. $\mathrm{R}_{f}: 0.46$ (PE/ AcOEt: $\left.70 / 30 \mathrm{v} / \mathrm{v}\right) . v_{\max } / \mathrm{cm}^{-1}$ $3615(\mathrm{NH}), 3362(\mathrm{OH}), 1596(\mathrm{~N}-\mathrm{H})$ and $1159(\mathrm{C}-\mathrm{OH}) .{ }^{1} \mathrm{H}$ NMR (300 MHz, DMSO-d6) $\delta 5.80$ (br s, 2H, NH $\left.\mathrm{NH}_{2}\right)$ 6.57-6.52 (m, 2H, H $\left.\mathrm{Ar}_{\mathrm{Ar}}\right), 6.65$ (s, 1H, $\left.\mathrm{H}_{\mathrm{Ar}}\right), 7.18-7.08\left(\mathrm{~m}, 1 \mathrm{H}, \mathrm{H}_{\mathrm{Ar}}\right), 7.29$ (d, $\left.J=1.4 \mathrm{~Hz}, 2 \mathrm{H}, \mathrm{H}_{\mathrm{Ar}}\right), 7.30\left(\mathrm{~s}, 2 \mathrm{H}, \mathrm{H}_{\mathrm{Ar}}\right), 7.61(\mathrm{~s}, 1 \mathrm{H}, \mathrm{OH}), 7.76\left(\mathrm{dd}, J=1.7,0.9,1 \mathrm{H}, \mathrm{H}_{\mathrm{Ar}}\right)$. ${ }^{13} \mathrm{C}$ NMR $(75 \mathrm{MHz}, \mathrm{DMSO}-\mathrm{d} 6) \delta 74.82\left(\mathrm{q},{ }^{2} J_{\mathrm{CF}}=30.4 \mathrm{~Hz}, \mathrm{C}-\mathrm{CF}_{3}\right), 109.21\left(\mathrm{CH}_{\mathrm{Ar}}\right), 110.42$ 
$\left(\mathrm{CH}_{\mathrm{Ar}}\right), 110.54\left(\mathrm{C}_{\mathrm{Ar}}\right), 122.60\left(\mathrm{CH}_{\mathrm{Ar}}\right), 122.78\left(\mathrm{C}_{\mathrm{Ar}}\right), 123.50\left(2 \mathrm{CH}_{\mathrm{Ar}}\right), 125.11\left(\mathrm{q},{ }^{1} J_{\mathrm{CF}}=286.1 \mathrm{~Hz}\right.$, $\left.\mathrm{CF}_{3}\right), 125.73\left(\mathrm{CH}_{\mathrm{Ar}}\right), 128.96\left(2 \mathrm{CH}_{\mathrm{Ar}}\right), 134.11\left(\mathrm{C}_{\mathrm{Ar}}\right), 143.60\left(\mathrm{CH}_{\mathrm{Ar}}\right), 150.91\left(\mathrm{C}_{\mathrm{Ar}}\right), 153.01\left(\mathrm{C}_{\mathrm{Ar}}\right)$. ${ }^{19}$ F NMR (376 MHz, DMSO-d6) $\delta-74.25\left(\mathrm{CF}_{3}\right)$. HPLC: $\mathrm{t}_{\mathrm{R}}: 30.28 \mathrm{~min}$. HRMS: $[\mathrm{M}+\mathrm{H}]^{+}$ calcd for $\mathrm{C}_{16} \mathrm{H}_{12} \mathrm{~F}_{3} \mathrm{NO}_{2} \mathrm{~S}$ : 339.0535; found: 339.0532 .

1-(2-amino-5-phenylthiophen-3-yl)-2,2,2-trifluoro-1-(thiophen-2-yl)ethan-1-ol (12d): Yield: 69\% (140 mg); brown powder: $\mathrm{mp}$ 120-121 ${ }^{\circ} \mathrm{C} . \mathrm{R}_{f}$ : 0.46 (PE/AcOEt: 70/30 v/v). $v_{\max } / \mathrm{cm}^{-1} 3619(\mathrm{NH}), 3244(\mathrm{OH}), 1606(\mathrm{~N}-\mathrm{H})$ and $1157(\mathrm{C}-\mathrm{OH}) .{ }^{1} \mathrm{H}$ NMR $(300 \mathrm{MHz}$, DMSO-d6) $\delta 5.52$ (br s, 2H, NH N $, 6.83\left(\mathrm{~s}, 1 \mathrm{H}, \mathrm{H}_{\mathrm{Ar}}\right), 7.04\left(\mathrm{t}, 1 \mathrm{H}, J=4.2 \mathrm{~Hz}, \mathrm{H}_{\mathrm{Ar}}\right), 7.10-7.16$ $\left(\mathrm{m}, 2 \mathrm{H}, \mathrm{H}_{\mathrm{Ar}}\right), 7.26-7.37\left(\mathrm{~m}, 5 \mathrm{H}, 4 \mathrm{H}_{\mathrm{Ar}}, \mathrm{OH}\right), 7.58\left(\mathrm{~d}, J=4.9 \mathrm{~Hz}, 1 \mathrm{H}, \mathrm{H}_{\mathrm{Ar}}\right) .{ }^{13} \mathrm{C} \mathrm{NMR}(75 \mathrm{MHz}$, DMSO-d6) $\delta 76.19\left(\mathrm{q},{ }^{2} J_{\mathrm{CF}}=27.7 \mathrm{~Hz}, \mathrm{C}-\mathrm{CF} 3\right), 113.09\left(\mathrm{CH}_{\mathrm{Ar}}\right), 122.08\left(\mathrm{CH}_{\mathrm{Ar}}\right), 122.89\left(\mathrm{CH}_{\mathrm{Ar}}\right)$, $123.57\left(2 \mathrm{CH}_{\mathrm{Ar}}\right), 125.79\left(\mathrm{CH}_{\mathrm{Ar}}\right), 126.05\left(\mathrm{q},{ }^{1} J_{\mathrm{CF}}=280.9 \mathrm{~Hz}, \mathrm{CF}_{3}\right), 126.41\left(\mathrm{CH}_{\mathrm{Ar}}\right), 126.75$ $\left(\mathrm{CH}_{\mathrm{Ar}}\right), 126.99\left(\mathrm{CH}_{\mathrm{Ar}}\right), 128.99\left(2 \mathrm{CH}_{\mathrm{Ar}}\right), 134.13\left(\mathrm{C}_{\mathrm{Ar}}\right), 143.25\left(\mathrm{C}_{\mathrm{Ar}}\right), 152.88\left(\mathrm{C}_{\mathrm{Ar}}\right) .{ }^{19} \mathrm{~F}$ NMR (376 MHz, DMSO-d6) $\delta-76.06\left(\mathrm{CF}_{3}\right)$. HPLC: $\mathrm{t}_{\mathrm{R}}: 32.27 \mathrm{~min}$. HRMS: $[\mathrm{M}+\mathrm{H}]^{+}$calcd for $\mathrm{C}_{16} \mathrm{H}_{13} \mathrm{~F}_{3} \mathrm{NOS}_{2}$ : 356.0391; found: 356.0397.

1-(2-amino-5-phenylthiophen-3-yl)-2,2,2-trifluoro-1-phenylethan-1-ol (12e): Yield: 82\% (163 mg); brown powder: $\mathrm{mp} 116-118^{\circ} \mathrm{C} . \mathrm{R}_{f}: 0.60$ (PE/AcOEt: $\left.70 / 30 \mathrm{v} / \mathrm{v}\right) . v_{\max } / \mathrm{cm}^{-1}$ $3376(\mathrm{NH}, \mathrm{OH}), 1608$ (N-H) and $1147(\mathrm{C}-\mathrm{OH}) .{ }^{1} \mathrm{H}$ NMR (300 MHz, DMSO-d6) $\delta 5.50$ (br $\left.\mathrm{s}, 2 \mathrm{H}, \mathrm{NH}_{2}\right), 6.95\left(\mathrm{~s}, 1 \mathrm{H}, \mathrm{H}_{\mathrm{Ar}}\right), 7.15\left(\mathrm{t}, J=7.1 \mathrm{~Hz}, 1 \mathrm{H}, \mathrm{H}_{\mathrm{Ar}}\right), 7.28-7.44\left(\mathrm{~m}, 8 \mathrm{H}, 7 \mathrm{H}_{\mathrm{Ar}}, \mathrm{OH}\right)$, $7.49\left(\mathrm{~d}, J=7.2 \mathrm{~Hz}, 2 \mathrm{H}, 2 \mathrm{H}_{\mathrm{Ar}}\right) .{ }^{13} \mathrm{C}$ NMR $(75 \mathrm{MHz}, \mathrm{DMSO}-\mathrm{d} 6) \delta 76.90\left(\mathrm{q},{ }^{2} J_{\mathrm{CF}}=27.9 \mathrm{~Hz}\right.$, $\left.\mathrm{C}-\mathrm{CF}_{3}\right), 114.35\left(\mathrm{C}_{\mathrm{Ar}}\right), 122.00\left(\mathrm{CH}_{\mathrm{Ar}}\right), 123.00\left(\mathrm{C}_{\mathrm{Ar}}\right), 123.63\left(2 \mathrm{CH}_{\mathrm{Ar}}\right), 125.67\left(\mathrm{q},{ }^{1} J_{\mathrm{CF}}=281.2 \mathrm{~Hz}\right.$, $\left.\mathrm{CF}_{3}\right), 125.79\left(\mathrm{CH}_{\mathrm{Ar}}\right), 127.16\left(2 \mathrm{CH}_{\mathrm{Ar}}\right), 128.02\left(2 \mathrm{CH}_{\mathrm{Ar}}\right), 128.35\left(\mathrm{CH}_{\mathrm{Ar}}\right), 129.06\left(2 \mathrm{CH}_{\mathrm{Ar}}\right), 134.30$ $\left(\mathrm{C}_{\mathrm{Ar}}\right), 138.80\left(\mathrm{C}_{\mathrm{Ar}}\right), 152.53\left(\mathrm{C}_{\mathrm{Ar}}\right) .{ }^{19} \mathrm{~F}$ NMR $(376 \mathrm{MHz}, \mathrm{DMSO}-\mathrm{d} 6) \delta-75.54\left(\mathrm{CF}_{3}\right)$. HPLC: $\mathrm{t}_{\mathrm{R}}$ : 32.93 min. HRMS: $[\mathrm{M}+\mathrm{H}]^{+}$calcd for $\mathrm{C}_{18} \mathrm{H}_{15} \mathrm{NOSF}_{3}, 350.0826$; found 350.0829.

1-(2-amino-5-phenylthiophen-3-yl)-2,2,2-trifluoro-1-(p-tolyl)ethan-1-ol (12f): Yield: 75\% (155 mg); brown powder: $\mathrm{mp} 113-115^{\circ} \mathrm{C} . \mathrm{R}_{f}$ : 0.56 (PE/ AcOEt: 70/30 v/v). $v_{\max } / \mathrm{cm}^{-1}$ $3628(\mathrm{NH}), 3336(\mathrm{OH}), 1610(\mathrm{~N}-\mathrm{H})$ and $1157(\mathrm{C}-\mathrm{OH}) .{ }^{1} \mathrm{H}$ NMR (300 MHz, DMSO-d6) $\delta$ $2.29\left(\mathrm{~s}, 3 \mathrm{H}, \mathrm{CH}_{3}\right), 5.48$ (br s, $\left.2 \mathrm{H}, \mathrm{NH}_{2}\right), 6.95$ (s, H, H $\left.\mathrm{Ar}_{\mathrm{Ar}}\right), 7.10-7.21\left(\mathrm{~m}, 3 \mathrm{H}, 3 \mathrm{H}_{\mathrm{Ar}}\right), 7.29-7.42$ $\left(\mathrm{m}, 7 \mathrm{H}, 6 \mathrm{H}_{\mathrm{Ar}}, \mathrm{OH}\right) .{ }^{13} \mathrm{C} \mathrm{NMR}(75 \mathrm{MHz}, \mathrm{DMSO}-\mathrm{d} 6) \delta 20.65\left(\mathrm{CH}_{3}\right), 76.78\left(\mathrm{q},{ }^{2} J_{\mathrm{CF}}=28.3 \mathrm{~Hz}\right.$, $\left.\mathrm{C}-\mathrm{CF}_{3}\right), 114.47\left(\mathrm{C}_{\mathrm{Ar}}\right), 122.00\left(\mathrm{C}_{\mathrm{Ar}}\right), 122.87\left(\mathrm{C}_{\mathrm{Ar}}\right), 123.57\left(2 \mathrm{CH}_{\mathrm{Ar}}\right), 125.70\left(\mathrm{CH}_{\mathrm{Ar}}\right), 126.96(\mathrm{q}$, $\left.{ }^{1} J_{\mathrm{CF}}=279.0 \mathrm{~Hz}, \mathrm{CF}_{3}\right), 127.07\left(2 \mathrm{CH}_{\mathrm{Ar}}\right), 128.54\left(2 \mathrm{CH}_{\mathrm{Ar}}\right), 129.00\left(2 \mathrm{CH}_{\mathrm{Ar}}\right), 134.30\left(\mathrm{C}_{\mathrm{Ar}}\right), 135.83$ $\left(\mathrm{C}_{\mathrm{Ar}}\right), 137.57\left(\mathrm{C}_{\mathrm{Ar}}\right), 152.46\left(\mathrm{C}_{\mathrm{Ar}}\right) .{ }^{19} \mathrm{~F}$ NMR $(376 \mathrm{MHz}, \mathrm{DMSO}-\mathrm{d} 6) \delta-76.22\left(\mathrm{CF}_{3}\right) . \mathrm{HPLC}: \mathrm{t}_{\mathrm{R}}$ : 34.05 min. HRMS: $[\mathrm{M}+\mathrm{H}]^{+}$calcd for $\mathrm{C}_{19} \mathrm{H}_{17} \mathrm{~F}_{3} \mathrm{NOS}$ : 346.0986; found: 364.0977 .

1-(2-amino-5-phenylthiophen-3-yl)-2,2,2-trifluoro-1-(4-fluorophenyl)ethan-1-ol (12g): Yield: $79 \%$ (165 mg); brown powder: $\mathrm{mp} 115-116{ }^{\circ} \mathrm{C} . \mathrm{R}_{f}$ : 0.46 (PE/AcOEt: 70/30 v/v). $v_{\max } / \mathrm{cm}^{-1} 3612(\mathrm{NH}), 3376(\mathrm{OH}), 1609(\mathrm{~N}-\mathrm{H})$ and $1144(\mathrm{C}-\mathrm{OH}) .{ }^{1} \mathrm{H}$ NMR $(300 \mathrm{MHz}, \mathrm{DMSO}-$ d6) $\delta 5.52\left(\right.$ br s, $\left.2 \mathrm{H}, \mathrm{NH}_{2}\right), 6.95-7.00\left(\mathrm{~m}, 1 \mathrm{H}, \mathrm{H}_{\mathrm{Ar}}\right), 7.11-7.18\left(\mathrm{~m}, 1 \mathrm{H}, \mathrm{H}_{\mathrm{Ar}}\right), 7.21(\mathrm{t}, \mathrm{J}=8.9 \mathrm{~Hz}$, $\left.2 \mathrm{H}, 2 \mathrm{H}_{\mathrm{Ar}}\right), 7.32\left(\mathrm{t}, J=7.7 \mathrm{~Hz}, 2 \mathrm{H}, 2 \mathrm{H}_{\mathrm{Ar}}\right), 7.37-7.42\left(\mathrm{~m}, 2 \mathrm{H}, 2 \mathrm{H}_{\mathrm{Ar}}\right), 7.44(\mathrm{~s}, 1 \mathrm{H}, \mathrm{OH}), 7.48-7.54$ $\left(\mathrm{m}, 2,2 \mathrm{H}_{\mathrm{Ar}}\right) .{ }^{13} \mathrm{C} \mathrm{NMR}(75 \mathrm{MHz}, \mathrm{DMSO}-\mathrm{d} 6) \delta 76.51\left(\mathrm{q},{ }^{2} J_{\mathrm{CF}}=28.7 \mathrm{~Hz}, \mathrm{C}-\mathrm{CF}_{3}\right), 114.05$ $\left(\mathrm{CH}_{\mathrm{Ar}}\right), 114.78\left(\mathrm{~d},{ }^{2} J_{\mathrm{CF}}=22.5 \mathrm{~Hz}, 2 \mathrm{CH}_{\mathrm{Ar}}\right), 121.75\left(\mathrm{CH}_{\mathrm{Ar}}\right), 123.07\left(\mathrm{C}_{\mathrm{Ar}}\right), 123.63\left(2 \mathrm{CH}_{\mathrm{Ar}}\right)$, $125.77\left(\mathrm{CH}_{\mathrm{Ar}}\right), 126.22\left(\mathrm{q},{ }^{1} J_{\mathrm{CF}}=286.5 \mathrm{~Hz}, \mathrm{CF}_{3}\right), 129.00\left(2 \mathrm{CH}_{\mathrm{Ar}}\right), 129.45\left(\mathrm{~d},{ }^{3} J_{\mathrm{CF}}=8.3 \mathrm{~Hz}\right.$, $\left.2 \mathrm{CH}_{\mathrm{Ar}}\right), 134.25\left(\mathrm{C}_{\mathrm{Ar}}\right), 134.88\left(\mathrm{C}_{\mathrm{Ar}}\right), 152.63\left(\mathrm{C}_{\mathrm{Ar}}\right), 161.96\left(\mathrm{~d},{ }^{1} J_{\mathrm{CF}}=243.0 \mathrm{~Hz}, \mathrm{C}_{\mathrm{Ar}}-\mathrm{F}\right) .{ }^{19} \mathrm{~F}$ NMR (376 MHz, DMSO-d6) $\delta-75.15\left(\mathrm{CF}_{3}\right),-114.21\left(\mathrm{C}_{\mathrm{Ar}}-\mathrm{F}\right)$. HPLC: $\mathrm{t}_{\mathrm{R}}:$ 30.28min. HRMS: $[\mathrm{M}+\mathrm{H}]^{+}$calcd for $\mathrm{C}_{18} \mathrm{H}_{14} \mathrm{~F}_{4} \mathrm{NOS}$ : 368.0727; found: 368.0725 .

1-(2-amino-5-phenylthiophen-3-yl)-1-(4-chlorophenyl)-2,2,2-trifluoroethan-1-ol (12h): Yield: 81\% (177 mg); brown powder: mp 120-122 ${ }^{\circ} \mathrm{C}$. $\mathrm{R}_{f}$ : 0.46 (PE/AcOEt: 70/30 v/v). $v_{\max } / \mathrm{cm}^{-1} 3601(\mathrm{NH}), 3349(\mathrm{OH}), 1596(\mathrm{~N}-\mathrm{H})$ and $1136(\mathrm{C}-\mathrm{OH}) .{ }^{1} \mathrm{H}$ NMR $(300 \mathrm{MHz}$, DMSO-d6) $\delta 5.53$ (br s, 2H, NH $\mathrm{NH}_{2}, 6.98\left(\mathrm{~s}, 1 \mathrm{H}, \mathrm{H}_{\mathrm{Ar}}\right), 7.15\left(\mathrm{t}, J=7.2 \mathrm{~Hz}, 1 \mathrm{H}, \mathrm{H}_{\mathrm{Ar}}\right), 7.32(\mathrm{t}$, $\left.J=7.7 \mathrm{~Hz}, 2 \mathrm{H}, 2 \mathrm{H}_{\mathrm{Ar}}\right), 7.38-7.42\left(\mathrm{~m}, 2 \mathrm{H}, 2 \mathrm{H}_{\mathrm{Ar}}\right), 7.45-7.51\left(\mathrm{~m}, 5 \mathrm{H}, 4 \mathrm{H}_{\mathrm{Ar}}, \mathrm{OH}\right) .{ }^{13} \mathrm{C} \mathrm{NMR}$ $(75 \mathrm{MHz}, \mathrm{DMSO}-\mathrm{d} 6) \delta 76.44\left(\mathrm{~d},{ }^{2} J_{\mathrm{CF}}=28.8 \mathrm{~Hz}, \mathrm{C}-\mathrm{CF}_{3}\right), 113.80\left(\mathrm{C}_{\mathrm{Ar}}\right), 121.67\left(\mathrm{CH}_{\mathrm{Ar}}\right), 123.11$ $\left(\mathrm{C}_{\mathrm{Ar}}\right), 123.63\left(2 \mathrm{CH}_{\mathrm{Ar}}\right), 123.92\left(\mathrm{q},{ }^{1} J_{\mathrm{CF}}=272.3 \mathrm{~Hz}, \mathrm{CF}_{3}\right), 125.79\left(\mathrm{CH}_{\mathrm{Ar}}\right), 127.99\left(2 \mathrm{CH}_{\mathrm{Ar}}\right), 129.00$ $\left(2 \mathrm{CH}_{\mathrm{Ar}}\right), 129.17\left(2 \mathrm{CH}_{\mathrm{Ar}}\right), 133.12\left(\mathrm{C}_{\mathrm{Ar}}\right), 134.21\left(\mathrm{C}_{\mathrm{Ar}}\right), 137.70\left(\mathrm{C}_{\mathrm{Ar}}\right), 152.68\left(\mathrm{C}_{\mathrm{Ar}}\right) .{ }^{19} \mathrm{~F} \mathrm{NMR}$ $(376 \mathrm{MHz}, \mathrm{DMSO}-\mathrm{d} 6) \delta-75.10\left(\mathrm{CF}_{3}\right)$. HPLC: $\mathrm{t}_{\mathrm{R}}: 30.28 \mathrm{~min}$. HRMS: $[\mathrm{M}+\mathrm{H}]^{+}$calcd for $\mathrm{C}_{18} \mathrm{H}_{14} \mathrm{ClF}_{3} \mathrm{NOS}$ : 384.0431; found: 384.0428 
1-(2-amino-5-phenylthiophen-3-yl)-1-(4-bromophenyl)-2,2,2-trifluoroethan-1-ol (12i): Yield: 81\% (198 mg); brown powder: $\mathrm{mp} 110-112{ }^{\circ} \mathrm{C} . \mathrm{R}_{f}$ : 0.46 (PE/AcOEt: 70/30 v/v). $v_{\max } / \mathrm{cm}^{-1} 3601(\mathrm{NH}), 3348(\mathrm{OH}), 1596(\mathrm{~N}-\mathrm{H})$ and $1135(\mathrm{C}-\mathrm{OH}) .{ }^{1} \mathrm{H}$ NMR $(300 \mathrm{MHz}$, DMSO-d6) $\delta 5.53$ (br s, 2H, NH 2 ), $6.98\left(\mathrm{~s}, 1 \mathrm{H}, \mathrm{H}_{\mathrm{Ar}}\right), 7.15\left(\mathrm{t}, J=7.2 \mathrm{~Hz}, 1 \mathrm{H}, \mathrm{H}_{\mathrm{Ar}}\right), 7.32(\mathrm{t}$, $\left.J=7.7 \mathrm{~Hz}, 2 \mathrm{H}, 2 \mathrm{H}_{\mathrm{Ar}}\right), 7.37-7.46\left(\mathrm{~m}, 4 \mathrm{H}, 4 \mathrm{H}_{\mathrm{Ar}}\right), 7.49(\mathrm{~s}, 1 \mathrm{H}, \mathrm{OH}), 7.55-7.63\left(\mathrm{~m}, 2 \mathrm{H}, 2 \mathrm{H}_{\mathrm{Ar}}\right)$. ${ }^{13} \mathrm{C}$ NMR $(75 \mathrm{MHz}, \mathrm{DMSO}-\mathrm{d} 6) \delta 76.55\left(\mathrm{q},{ }^{2} \mathrm{~J}_{\mathrm{CF}}=28.52 \mathrm{~Hz}, \mathrm{C}-\mathrm{CF}_{3}\right), 113.78\left(\mathrm{C}_{\mathrm{Ar}}\right), 121.65$ $\left(\mathrm{CH}_{\mathrm{Ar}}\right), 121.83\left(\mathrm{C}_{\mathrm{Ar}}\right), 123.14\left(\mathrm{C}_{\mathrm{Ar}}\right), 123.63\left(2 \mathrm{CH}_{\mathrm{Ar}}\right), 125.29\left(\mathrm{q},{ }^{1} \mathrm{~J}_{\mathrm{CF}}=285.6 \mathrm{~Hz}, \mathrm{CF}_{3}\right) 125.77$ $\left(\mathrm{CH}_{\mathrm{Ar}}\right), 128.98\left(2 \mathrm{CH}_{\mathrm{Ar}}\right), 129.48\left(2 \mathrm{CH}_{\mathrm{Ar}}\right), 130.91\left(2 \mathrm{CH}_{\mathrm{Ar}}\right), 134.21\left(\mathrm{C}_{\mathrm{Ar}}\right), 138.14\left(\mathrm{C}_{\mathrm{Ar}}\right), 152.69$ $\left(\mathrm{C}_{\mathrm{Ar}}\right) .{ }^{19} \mathrm{~F}$ NMR $(376 \mathrm{MHz}, \mathrm{DMSO}-\mathrm{d} 6) \delta-75.10\left(\mathrm{CF}_{3}\right)$. HPLC: $\mathrm{t}_{\mathrm{R}}: 30.28 \mathrm{~min}$. HRMS: $[\mathrm{M}+\mathrm{H}]^{+}$calcd for $\mathrm{C}_{18} \mathrm{H}_{14} \mathrm{BrF}_{3} \mathrm{NOS}$ : 427.9926; found: 427.9917.

1-(2-amino-5-phenylthiophen-3-yl)-2,2,2-trifluoro-1-(pyridin-3-yl)ethan-1-ol (12j): Yield: 93\% (185 mg); brown crystals: mp 121-122 ${ }^{\circ} \mathrm{C}$. $\mathrm{R}_{f}$ : 0.26 (PE/AcOEt: 80/20 v/v). $v_{\max } / \mathrm{cm}^{-1}$ $3599(\mathrm{NH}), 3389(\mathrm{OH})$ and $1169(\mathrm{C}-\mathrm{OH}) .{ }^{1} \mathrm{H}$ NMR $(300 \mathrm{MHz}, \mathrm{DMSO}-\mathrm{d} 6) \delta 5.78$ (br s, 2H, $\left.\mathrm{NH}_{2}\right), 6.95\left(\mathrm{~s}, 1 \mathrm{H}, \mathrm{H}_{\mathrm{Ar}}\right), 7.13\left(\mathrm{t}, J=6.7 \mathrm{~Hz}, 1 \mathrm{H}, \mathrm{H}_{\mathrm{Ar}}\right), 7.24-7.38\left(\mathrm{~m}, 4 \mathrm{H}, 4 \mathrm{H}_{\mathrm{Ar}}\right), 7.39-7.45(\mathrm{~m}$, $\left.1 \mathrm{H}, \mathrm{H}_{\mathrm{Ar}}\right), 7.47(\mathrm{~s}, 1 \mathrm{H}, \mathrm{OH}), 7.69\left(\mathrm{~d}, J=8.1 \mathrm{~Hz}, 1 \mathrm{H}, \mathrm{H}_{\mathrm{Ar}}\right), 7.90\left(\mathrm{t}, J=7.8 \mathrm{~Hz}, 1 \mathrm{H}, \mathrm{H}_{\mathrm{Ar}}\right), 8.62(\mathrm{~d}$, $\left.J=4.7 \mathrm{~Hz}, 1 \mathrm{H}, \mathrm{H}_{\mathrm{Ar}}\right) .{ }^{13} \mathrm{C} \mathrm{NMR}(75 \mathrm{MHz}, \mathrm{DMSO}-\mathrm{d} 6) \delta 77.97\left(\mathrm{q},{ }^{2} J_{\mathrm{CF}}=28.3 \mathrm{~Hz}, \mathrm{C}-\mathrm{CF}_{3}\right)$, $112.69\left(\mathrm{C}_{\mathrm{Ar}}\right), 122.25\left(\mathrm{CH}_{\mathrm{Ar}}\right), 122.59\left(\mathrm{CH}_{\mathrm{Ar}}\right), 122.95\left(\mathrm{C}_{\mathrm{Ar}}\right), 123.52\left(2 \mathrm{CH}_{\mathrm{Ar}}\right), 123.68\left(\mathrm{CH}_{\mathrm{Ar}}\right)$, $125.64\left(\mathrm{q},{ }^{1} J_{\mathrm{CF}}=276.4 \mathrm{~Hz}, \mathrm{CF}_{3}\right), 125.71\left(\mathrm{CH}_{\mathrm{Ar}}\right), 128.97\left(2 \mathrm{CH}_{\mathrm{Ar}}\right), 134.22\left(\mathrm{C}_{\mathrm{Ar}}\right), 137.46\left(\mathrm{CH}_{\mathrm{Ar}}\right)$, $147.72\left(\mathrm{CH}_{\mathrm{Ar}}\right), 152.53\left(\mathrm{C}_{\mathrm{Ar}}\right), 157.48\left(\mathrm{C}_{\mathrm{Ar}}\right) .{ }^{19} \mathrm{~F}$ NMR $(376 \mathrm{MHz}, \mathrm{DMSO}-\mathrm{d} 6) \delta-74.25\left(\mathrm{CF}_{3}\right)$. HPLC: $t_{R}$ : 31.37 min. HRMS: $[\mathrm{M}+\mathrm{H}]^{+}$calcd for $\mathrm{C}_{17} \mathrm{H}_{14} \mathrm{~N}_{2} \mathrm{OF}_{3} \mathrm{~S}$ : 351.0779; found: 351.0793 .

1-(2-amino-5-phenylthiophen-3-yl)-1-(benzo[d]thiazol-2-yl)-2,2,2-trifluoroethan-1-ol (12k): Yield: 80\% (185 mg); brown powder: $\mathrm{mp} 115-117^{\circ} \mathrm{C} . \mathrm{R}_{f}$ : 0.46 (PE/AcOEt: 70/30 v/v). $v_{\max } / \mathrm{cm}^{-1} 3606(\mathrm{NH}), 3376(\mathrm{OH}), 1609(\mathrm{~N}-\mathrm{H})$ and $1157(\mathrm{C}-\mathrm{OH}) .{ }^{1} \mathrm{H}$ NMR $(300 \mathrm{MHz}$, DMSO-d6) $\delta 6.01$ (br s, 2H, NH NH $_{2}, 7.08\left(\mathrm{~s}, 1 \mathrm{H}, \mathrm{H}_{\mathrm{Ar}}\right), 7.10-7.17\left(\mathrm{~m}, 1 \mathrm{H}, \mathrm{H}_{\mathrm{Ar}}\right), 7.26-7.36(\mathrm{~m}$, $\left.4 \mathrm{H}, 4 \mathrm{H}_{\mathrm{Ar}}\right), 7.47-7.60\left(\mathrm{~m}, 2 \mathrm{H}, 2 \mathrm{H}_{\mathrm{Ar}}\right), 8.08-8.18\left(\mathrm{~m}, 2 \mathrm{H}, 2 \mathrm{H}_{\mathrm{Ar}}\right) 8.64(\mathrm{~s}, 1 \mathrm{H}, \mathrm{OH}) .{ }^{13} \mathrm{C} \mathrm{NMR}$ $(75 \mathrm{MHz}, \mathrm{DMSO}-\mathrm{d} 6) \delta 77.60\left(\mathrm{q},{ }^{2} J_{\mathrm{CF}}=29.9 \mathrm{~Hz}, \mathrm{C}-\mathrm{CF}_{3}\right), 110.61\left(\mathrm{C}_{\mathrm{Ar}}\right), 122.23\left(\mathrm{CH}_{\mathrm{Ar}}\right), 122.35$ $\left(\mathrm{CH}_{\mathrm{Ar}}\right), 123.38\left(\mathrm{CH}_{\mathrm{Ar}}\right), 123.59\left(2 \mathrm{CH}_{\mathrm{Ar}}\right), 124.66\left(\mathrm{q},{ }^{1} J_{\mathrm{CF}}=287.8 \mathrm{~Hz}, \mathrm{CF}_{3}\right), 125.87\left(\mathrm{CH}_{\mathrm{Ar}}\right)$, $125.90\left(\mathrm{CH}_{\mathrm{Ar}}\right), 126.47\left(\mathrm{CH}_{\mathrm{Ar}}\right), 128.99\left(2 \mathrm{CH}_{\mathrm{Ar}}\right), 133.96\left(\mathrm{C}_{\mathrm{Ar}}\right), 134.67\left(\mathrm{C}_{\mathrm{Ar}}\right), 152.48\left(\mathrm{C}_{\mathrm{Ar}}\right)$, $153.15\left(2 \mathrm{C}_{\mathrm{Ar}}\right), 171.32\left(\mathrm{C}_{\mathrm{Ar}}\right) .{ }^{19} \mathrm{~F}$ NMR $(376 \mathrm{MHz}, \mathrm{DMSO}-\mathrm{d} 6) \delta-75.14\left(\mathrm{CF}_{3}\right)$. HPLC: $\mathrm{t}_{\mathrm{R}}$ : 30.28 min. HRMS: $[\mathrm{M}+\mathrm{H}]^{+}$calcd for $\mathrm{C}_{19} \mathrm{H}_{14} \mathrm{~F}_{3} \mathrm{~N}_{2} \mathrm{OS}_{2}$ : 407.0494; found: 407.0500.

1-(2-amino-5-phenylthiophen-3-yl)-2,2,2-trifluoro-1-(naphthalen-2-yl)ethan-1-ol (12l): Yield: $72 \%$ (164 mg); brown powder: $\mathrm{mp} 127-128^{\circ} \mathrm{C} . \mathrm{R}_{f}: 0.60$ (PE/AcOEt: 70/30 v/v). $v_{\max } / \mathrm{cm}^{-1} 3575(\mathrm{NH}), 3336(\mathrm{OH}), 1609(\mathrm{~N}-\mathrm{H})$ and $1134(\mathrm{C}-\mathrm{OH}) .{ }^{1} \mathrm{H}$ NMR $(300 \mathrm{MHz}$, DMSO-d6) $\delta 5.84$ (br s, 2H, $\left.\mathrm{NH}_{2}\right), 6.37$ (s, 1H, $\left.\mathrm{H}_{\mathrm{Ar}}\right), 6.99-7.34\left(\mathrm{~m}, 5 \mathrm{H}, 5 \mathrm{H}_{\mathrm{Ar}}\right), 7.35-7.50$ $\left(\mathrm{m}, 2 \mathrm{H}, 2 \mathrm{H}_{\mathrm{Ar}}\right), 7.57\left(\mathrm{t}, J=7.8 \mathrm{~Hz}, 1 \mathrm{H}, \mathrm{H}_{\mathrm{Ar}}\right), 7.74\left(\mathrm{~s}, 1 \mathrm{H}, \mathrm{H}_{\mathrm{Ar}}\right), 7.77(\mathrm{br} \mathrm{s}, 1 \mathrm{H}, \mathrm{OH}), 7.93(\mathrm{~d}$, $\left.J=7.2 \mathrm{~Hz}, 1 \mathrm{H}, \mathrm{H}_{\mathrm{Ar}}\right), 7.99\left(\mathrm{~d}, J=8.4 \mathrm{~Hz}, 1 \mathrm{H}, \mathrm{H}_{\mathrm{Ar}}\right), 8.43\left(\mathrm{~d}, J=8.6 \mathrm{~Hz}, 1 \mathrm{H}, \mathrm{H}_{\mathrm{Ar}}\right) .{ }^{13} \mathrm{C} \mathrm{NMR}$ $(75 \mathrm{MHz}, \mathrm{DMSO}-\mathrm{d} 6) \delta 79.47\left(\mathrm{q},{ }^{2} J_{\mathrm{CF}}=26.9 \mathrm{~Hz}\right), 113.04\left(\mathrm{C}_{\mathrm{Ar}}\right), 122.88\left(\mathrm{CH}_{\mathrm{Ar}}\right), 122.97\left(\mathrm{CH}_{\mathrm{Ar}}\right)$, $123.33\left(2 \mathrm{CH}_{\mathrm{Ar}}\right), 123.42\left(\mathrm{C}_{\mathrm{Ar}}\right), 124.54\left(\mathrm{CH}_{\mathrm{AR}}\right), 125.61\left(3 \mathrm{CH}_{\mathrm{Ar}}\right), 126.21\left(\mathrm{q},{ }^{1} J_{\mathrm{CF}}=281.2 \mathrm{~Hz}, \mathrm{CF}_{3}\right)$, $126.86\left(\mathrm{CH}_{\mathrm{Ar}}\right), 128.74\left(\mathrm{CH}_{\mathrm{Ar}}\right), 128.90\left(2 \mathrm{CH}_{\mathrm{Ar}}\right), 129.83\left(\mathrm{CH}_{\mathrm{Ar}}\right), 130.90\left(\mathrm{C}_{\mathrm{Ar}}\right), 133.96\left(\mathrm{C}_{\mathrm{Ar}}\right), 134.41$ $\left(\mathrm{C}_{\mathrm{Ar}}\right), 134.52\left(\mathrm{C}_{\mathrm{Ar}}\right), 151.68\left(\mathrm{C}_{\mathrm{Ar}}\right) .{ }^{19} \mathrm{~F}$ NMR $(376 \mathrm{MHz}, \mathrm{DMSO}-\mathrm{d} 6) \delta-74.25\left(\mathrm{CF}_{3}\right)$. HPLC: $\mathrm{t}_{\mathrm{R}}$ : 33.70 min. HRMS: $[\mathrm{M}+\mathrm{H}]^{+}$calcd for $\mathrm{C}_{22} \mathrm{H}_{17} \mathrm{~F}_{3} \mathrm{NOS}$ : 400.0977; found: 400.095 .

\subsection{Crystallographic Data}

CCDC 2083160 contains the supplementary crystallographic data for this paper. These data can be obtained free of charge via http:/ / www.ccdc.cam.ac.uk/conts / retrieving.html (or from the CCDC, 12 Union Road, Cambridge CB2 1EZ, UK; Fax: +44 1223 336033; E-mail: deposit@ccdc.cam.ac.uk)

Supplementary Materials: The following are available online, Figure S1: ${ }^{1} \mathrm{H}$ spectra of 9 in $\mathrm{D}_{2} \mathrm{O}$ (singlet at $7.28 \mathrm{ppm} ; 300 \mathrm{MHz}$ ); Figure S2: ${ }^{13} \mathrm{C}$ spectra of 9 in $\mathrm{D}_{2} \mathrm{O}$ (triplet at $123.40 \mathrm{ppm}$; $75 \mathrm{MHz}$ ); Figure S3: Crystal structure of compounds $\mathbf{1 2} \mathbf{j}$; Figure S4: (A) XP diagram of compound $\mathbf{1 2} \mathbf{j}$ with atomic numbering scheme; (B) Expandation of the packing diagram of compound 12j within the crystal mesh trough intra and intermolecular hydrogen bonds; Table S1: Optimization attempts for the synthesis of 16-17; Table S2: Optimization studies for the synthesis of 12e; Table S3: Optimization 
attempts for the synthesis of 13; Table S4: Kinetics considerations following HPLC spectra; Table S5: Crystal data and structure refinement details for $\mathbf{1 2} \mathbf{j}$; Table S6: Bond lengths for $\mathbf{1 2} \mathbf{j}(\AA)$; Table S7: Bond angles for $\mathbf{1 2} \mathbf{j}\left({ }^{\circ}\right)$; Table S8: Torsion angles for $\mathbf{1 2} \mathbf{j}\left({ }^{\circ}\right)$; Table S9: Hydrogen bond distances $(\AA)$ and angles for $\mathbf{1 2 j}\left({ }^{\circ}\right)$. Characterization of compounds $\left({ }^{1} \mathrm{H} 300 \mathrm{MHz},{ }^{13} \mathrm{C} 75 \mathrm{MHz},{ }^{19} \mathrm{~F}\right.$ NMR $376 \mathrm{MHz}$, DEPT-135 in DMSO-d 6 ; HRMS, HPLC).

Author Contributions: Conceptualization, Z.B., P.M. and V.D.; methodology, V.D. and D.B.; investigation, V.D. and D.B.; Resources: V.D.; data curation, V.D.; writing-original draft preparation, V.D.; writing-review and editing, V.D., P.M. and Z.B.; supervision, Z.B.; project administration, Z.B. All authors have read and agreed to the published version of the manuscript.

Funding: This research received no external funding.

Institutional Review Board Statement: Not applicable.

Informed Consent Statement: Not applicable.

Data Availability Statement: Not applicable.

Acknowledgments: We gratefully thank the French Ministère de l'Enseignement Supérieur, de la Recherche et de l'Innovation (MESRI). The authors also thank the University of Nîmes and the Occitanie Region for the financial support.

Conflicts of Interest: The authors declare no conflict of interest.

Sample Availability: Samples of the compounds are available from the authors.

\section{References}

1. Bozorov, K.; Nie, L.F.; Zhao, J.; Aisa, H.A. 2-Aminothiophene scaffolds: Diverse biological and pharmacological attributes in medicinal chemistry. Eur. J. Med. Chem. 2017, 140, 465-493. [CrossRef]

2. Thanna, S.; Knudson, S.E.; Grzegorzewicz, A.; Kapil, S.; Goins, C.M.; Ronning, D.R.; Jackson, M.; Slayden, R.A.; Sucheck, S.J. Synthesis and evaluation of new 2-aminothiophenes against: Mycobacterium tuberculosis. Org. Biomol. Chem. 2016, 14, 6119-6133. [CrossRef]

3. Scheich, C.; Puetter, V.; Schade, M. Novel small molecule inhibitors of MDR mycobacterium tuberculosis by NMR fragment screening of antigen 85C. J. Med. Chem. 2010, 53, 8362-8367. [CrossRef]

4. Oza, V.; Ashwell, S.; Almeida, L.; Brassil, P.; Breed, J.; Deng, C.; Gero, T.; Grondine, M.; Horn, C.; Ioannidis, S.; et al. Discovery of checkpoint kinase inhibitor (S)-5-(3-fluorophenyl)-N-(piperidin-3-yl)-3-ureidothiophene-2-carboxamide (AZD7762) by structurebased design and optimization of thiophenecarboxamide ureas. J. Med. Chem. 2012, 55, 5130-5142. [CrossRef]

5. Desantis, J.; Nannetti, G.; Massari, S.; Barreca, M.L.; Manfroni, G.; Cecchetti, V.; Palù, G.; Goracci, L.; Loregian, A.; Tabarrini, O Exploring the cycloheptathiophene-3-carboxamide scaffold to disrupt the interactions of the influenza polymerase subunits and obtain potent anti-influenza activity. Eur. J. Med. Chem. 2017, 138, 128-139. [CrossRef]

6. Narlawar, R.; Lane, J.R.; Doddareddy, M.; Lin, J.; Brussee, J.; Ijzerman, A.P. Hybrid ortho/allosteric ligands for the adenosine A1 receptor. J. Med. Chem. 2010, 53, 3028-3037. [CrossRef]

7. Tang, J.; Huber, A.D.; Pineda, D.L.; Boschert, K.N.; Wolf, J.J.; Kankanala, J.; Xie, J.; Sarafianos, S.G.; Wang, Z. 5-Aminothiophene2,4-dicarboxamide analogues as hepatitis B virus capsid assembly effectors. Eur. J. Med. Chem. 2019, 164, 179-192. [CrossRef]

8. Aly, H.M.; Saleh, N.M.; Elhady, H.A. Design and synthesis of some new thiophene, thienopyrimidine and thienothiadiazine derivatives of antipyrine as potential antimicrobial agents. Eur. J. Med. Chem. 2011, 46, 4566-4572. [CrossRef]

9. Campaigne, E.; Foye, W.O. The synthesis of 2,5-diarylthiophenes. J. Org. Chem. 1952, 17, 1405-1412. [CrossRef]

10. Gewald, K. Heterocyclen aus $\mathrm{CH}$-aciden Nitrilen, VIII. 2-Amino-thiophene aus methylenaktiven Nitrilen; Carbonylverbindungen und Schwefel. Chem. Ber. 1965, 98, 3571-3577. [CrossRef]

11. Minetto, G.; Raveglia, L.F.; Sega, A.; Taddei, M. Microwave-assisted Paal-Knorr reaction-Three-step regiocontrolled synthesis of polysubstituted furans, pyrroles and thiophenes. Eur. J. Org. Chem. 2005, 2005, 5277-5288. [CrossRef]

12. Revelant, G.; Dunand, S.; Hesse, S.; Kirsch, G. Microwave-assisted synthesis of 5-substituted 2-aminothiophenes starting from arylacetaldehydes. Synthesis 2011, 2011, 2935-2940. [CrossRef]

13. Gouda, M.A.; Al-Ghorbani, M.; Al-Zaqri, N. Synthesis and cytotoxic activity of some new heterocycles incorporating cyclohepta[b]thiophene-3-carboxamide derivatives. J. Heterocycl. Chem. 2020, 57, 3664-3672. [CrossRef]

14. Ibrahim, B.A.; Mohareb, R.M. Uses of ethyl benzoyl acetate for the synthesis of thiophene, pyran, and pyridine derivatives with antitumor activities. J. Heterocycl. Chem. 2020, 57, 4023-4035. [CrossRef]

15. Hwang, J.; Borgelt, L.; Wu, P. Multicomponent petasis reaction for the synthesis of functionalized 2-aminothiophenes and thienodiazepines. ACS Comb. Sci. 2020, 22, 495-499. [CrossRef]

16. Touré, B.B.; Hall, D.G. Natural product synthesis using multicomponent reaction strategies. Chem. Rev. 2009, 109, 4439-4486. [CrossRef] 
17. Bauer, I.; Knölker, H.J. Iron catalysis in organic synthesis. Chem. Rev. 2015, 115, 3170-3387. [CrossRef]

18. Poulsen, T.B.; Jørgensen, K.A. Catalytic asymmetric Friedel-Crafts alkylation reactions-Copper showed the way. Chem. Rev. 2008, 108, 2903-2915. [CrossRef]

19. Nie, J.; Guo, H.C.; Cahard, D.; Ma, J.A. Asymmetric construction of stereogenic carbon centers featuring a trifluoromethyl group from prochiral trifluoromethylated substrates. Chem. Rev. 2011, 111, 455-529. [CrossRef]

20. Fuson, R.C.; Weinstock, H.H.; Ullyot, G.E. A new synthesis of benzoins. $2^{\prime}, 4^{\prime}, 6^{\prime}$-trimethylbenzoin. J. Am. Chem. Soc. 1935, 57, 1803-1804. [CrossRef]

21. Pillaiyar, T.; Sedaghati, M.; Schnakenburg, G. Reaction of indoles with aromatic fluoromethyl ketones: An efficient synthesis of trifluoromethyl(indolyl)phenylmethanols using $\mathrm{K} 2 \mathrm{CO} 3 / \mathrm{n}-\mathrm{Bu} 4 \mathrm{PBr}$ in water. Beilstein J. Org. Chem. 2020, 16, 778-7490. [CrossRef]

22. Harikrishnan, A.; Selvakumar, J.; Gnanamani, E.; Bhattacharya, S.; Ramanathan, C.R. Friedel-Crafts hydroxyalkylation through activation of a carbonyl group using AlBr3: An easy access to pyridyl aryl/heteroaryl carbinols. New J. Chem. 2013, 37, 563-567. [CrossRef]

23. Medimagh, R.; Marque, S.; Prim, D.; Chatti, S. A facile preparation of trisubstituted amino-furan and -thiophene derivatives. Org. Biomol. Chem. 2011, 9, 6055-6065. [CrossRef]

24. Purser, S.; Moore, P.R.; Swallow, S.; Gouverneur, V. Fluorine in medicinal chemistry. Chem. Soc. Rev. 2008, 37, 320-330. [CrossRef]

25. Umemoto, T. Electrophilic perfluoroalkylating agents. Chem. Rev. 1996, 96, 1757-1777. [CrossRef]

26. Prakash, G.K.S.; Yudin, A.K. Perfluoroalkylation with organosilicon reagents. Chem. Rev. 1997, 97, 757-786. [CrossRef]

27. Kirij, N.V.; Yagupolskii, Y.L.; Petukh, N.V.; Tyrra, W.; Naumann, D. Trifluoromethylation of heterocumulenes with trimethyl(trifluoromethyl)silane in the presence of fluoride ions: Synthesis of trifluoroacetamides and trifluorothioacetamides from isocyanates and isothiocyanates. Tetrahedron Lett. 2001, 42, 8181-8183. [CrossRef]

28. Horváth, I.T.; Anastas, P.T. Innovations and green chemistry. Chem. Rev. 2007, 107, 2169-2173. [CrossRef]

29. Trost, B.M. On inventing reactions for atom economy. Acc. Chem. Res. 2002, 35, 695-705. [CrossRef]

30. Trost, B.M. Atom economy-A challenge for organic synthesis: Homogeneous catalysis leads the way. Angew. Chem. Int. Ed. Engl. 1995, 34, 259-281. [CrossRef]

31. Anastas, P.; Eghbali, N. Green chemistry: Principles and practice. Chem. Soc. Rev. 2009, 39, 301-312. [CrossRef] [PubMed]

32. Boibessot, T.; Zschiedrich, C.P.; Dunyach-Rémy, C.; Lebeau, A.; Bénimèlis, D.; Dunyach-Rémy, C.; Lavigne, J.-P.; Szurmant, H.; Benfodda, Z.; Meffre, P. The rational design, synthesis, and antimicrobial properties of thiophene derivatives that inhibit bacterial histidine kinases. J. Med. Chem. 2016, 59, 8830-8847. [CrossRef] [PubMed]

33. Nguyen, T.; Gamage, T.F.; Decker, A.M.; Barrus, D.; Langston, T.L.; Li, J.-X.; Thomas, B.F.; Zhang, Y. Synthesis and pharmacological evaluation of 1-phenyl-3-thiophenylurea derivatives as cannabinoid type-1 receptor allosteric modulators. J. Med. Chem. 2019, 62, 9806-9823. [CrossRef] [PubMed]

34. Garnett, J.L.; Sollich-Baumgartner, W.A. Catalytic deuterium exchange reactions with organics. XIV. Distinction between associative and dissociative $\pi$-complex substitution mechanisms. J. Phys. Chem. 1964, 68, 3177-3183. [CrossRef]

35. Khodakovskiy, P.V.; Mykhailiuk, P.K.; Volochnyuk, D.M.; Tolmachev, A.A. Noncatalytic electrophilic oxyalkylation of some five-membered heterocycles with 2-(trifluoroacetyl)-1,3-azoles. Synthesis 2010, 979-984. [CrossRef]

36. Nenajdenko, V.G.; Sanin, A.V.; Balenkova, E.S. Preparation of $\alpha, \beta$-unsaturated ketones bearing a trifluoromethyl group and their application in organic synthesis. Molecules 1997, 2, 186-232. [CrossRef]

37. Ho, T.L. The hard soft acids bases (HSAB) principle and organic chemistry. Chem. Rev. 1975, 75, 1-20. [CrossRef]

38. Davies, H.M.L.; Hedley, S.J. Intermolecular reactions of electron-rich heterocycles with copper and rhodium carbenoids. Chem. Soc. Rev. 2007, 36, 1109-1119. [CrossRef]

39. Smith, M.B. March's Advanced Organic Chemistry, 7th ed.; John Wiley \& Sons, Ltd.: Hoboken, NJ, USA, 2013; ISBN 978-0-470-46259-1.

40. Li, C.J. Organic reactions in aqueous media with a focus on carbon-carbon bond formations: A decade update. Chem. Rev. 2005, 105, 3095-3165. [CrossRef]

41. Ding, R.; Zhang, H.B.; Chen, Y.J.; Liu, L.; Wang, D.; Li, C.J. In(OTf)3-catalyzed Friedel-Crafts reaction of aromatic compounds with methyl trifluoropyruvate in water. Synlett 2004, 3, 555-557. [CrossRef] 